\title{
Stabilised finite element methods for the Oseen problem on anisotropic quadrilateral meshes*
}

\author{
GABRIEL R. BARRENECHEA ${ }^{\dagger}$ \\ Department of Mathematics and Statistics, \\ University of Strathclyde, \\ 26 Richmond Street, Glasgow G1 1XH, Scotland \\ AND \\ ANDREAS WACHTEL ${ }^{\ddagger}$ \\ Departamento académico de Matématicas, \\ ITAM - Instituto Tecnológico Autónomo de México
}

June 20, 2017

\begin{abstract}
In this work we present and analyse new inf-sup stable, and stabilised, finite element methods for the Oseen equation in anisotropic quadrilateral meshes. The meshes are formed of closed parallelograms, and the analysis is restricted to two space dimensions. Starting with the lowest order $\mathbb{Q}_{1}^{2} \times \mathbb{P}_{0}$ pair, we first identify the pressure components that make this finite element pair to be non-inf-sup stable, especially with respect to the aspect ratio. We then propose a way to penalise them, both strongly, by directly removing them from the space, and weakly, by adding a stabilisation term based on jumps of the pressure across selected edges. Concerning the velocity stabilisation, we propose an enhanced grad-div term. Stability and optimal a priori error estimates are given, and the results are confirmed numerically.

Keywords: Oseen equation, stabilised finite element method, anisotropic quadrilateral mesh
\end{abstract}

\section{Introduction}

In this paper we discuss stabilised finite element methods for the Oseen problem on highly anisotropic meshes. This equation appears, for example, in the iterative solution

*This research was supported by the Leverhulme Trust under grant RPG-2012-483.

${ }^{\dagger}$ gabriel.barrenechea@strath.ac.uk

${ }^{\ddagger}$ andreas.wachtel@itam.mx 
of the Navier-Stokes equations. As it is the case with the Navier-Stokes equation, when solving the Oseen problem numerically three different aspects can affect the quality of the numerical solution, and then need to be treated. One is a compatibility condition between velocity and pressure spaces, namely the inf-sup condition, that requires to be satisfied, or circumvented. In addition, if the mesh is not refined enough, then the local Péclet number is much larger than one (i.e., if the problem is convection-dominated), then the numerical solution usually presents spurious, non physical, oscillations. Finally, even if the solution to the continuous equation is divergence-free, usually the discrete solution is not. This can affect (sometimes dramatically) the quality of the numerical solution, especially when the Navier-Stokes equations are coupled to, say, temperature equations.

We start by discussing the first restriction mentioned in the previous paragraph. The first approach was to design pairs of velocity and pressure spaces that satisfy the inf-sup condition. For extensive reviews of such an approach on shape-regular meshes we refer to [GR86, BBF13], and the references therein. Now, the simplest finite element pairs, namely, equal-order interpolation for velocity and pressure, or the $\mathbb{Q}_{1}^{2} \times \mathbb{P}_{0}$ element, are not inf-sup stable. Then, in the mid eighties the idea of stabilisation appeared in order to circumvent this restriction. Examples of stabilised methods are PSPG methods, introduced for shape-regular meshes in [HFB86], and extended to anisotropic meshes in [MPP03, Bla08]. Later on, different approaches have been proposed to stabilise this restriction, including Residual-Free Bubbles, or enriched finite element methods (see, e.g., [BBF93, ABV06]), and Local Projection Stabilised (LPS) methods (see [BB01]). Alternatively, and as an attempt to analyse some of these methods in a unified manner, the idea of minimal stabilisation was proposed in [BF01]. This approach consists of splitting the pressure space into the sum of a stable part and an unstable part. Then, a stabilising term is added to the formulation to control the unstable part of the pressure space, hence restoring stability. This approach provided a different interpretation of some older methods, e.g., [PS85], the local jumps stabilisation [KS92], and pressure projection [BDG06] (see [Bur08] for a unified presentation of this idea). In addition, this approach was recently used to design new inf-sup stable, and stabilised, finite element methods for the Stokes problem on anisotropic meshes in [ABW15] (modifying a decomposition given previously in [AC00]). This last work concerned for the pairs $\mathbb{Q}_{k+1}^{2} \times \mathbb{P}_{k-1}^{2}, k \geq 1$, and then does not cover the lowest order case. For the latter case, and in the context of the Stokes problem, the local jump method from [KS92] was recently extended to anisotropic meshes without corner patches in [LS13], and to meshes containing corner patches in [BW15].

Regarding the second source of instability, namely, the presence of a dominating convection, one of the earliest approaches is the Streamline Upwind Petrov-Galerkin (SUPG) method, introduced in [BH82] for shape-regular meshes. The main idea of this method has been later on revisited in, e.g., [HFH89, FF92], and extended to a class of anisotropic meshes in [AKL08]. For the Oseen (and Navier-Stokes) equations, both GLS and SUPG methods introduce additional (unphysical) coupling terms between velocity and pressure. On the other hand, their stability is only due to the symmetric, diagonal, terms appearing in their definition. This observation motivated the extension of LPS methods for convection dominated problems, see for example [MST07, Kno10, MT15], or [RST08, BB+07] for overviews. For anisotropic meshes, up to our best knowledge, the only work concerning the LPS method on anisotropic meshes is the work [Bra08], 
where the method is applied to the Oseen and Navier-Stokes equations using equal order $\mathbb{Q}_{1}^{2} \times \mathbb{Q}_{1}$ elements on anisotropic, structured, quadrilateral meshes.

Concerning the satisfaction of the divergence-free character of the velocity field at the discrete level, the numerical velocities obtained using inf-sup stable elements are discretely divergence-free by definition, although this might not be enough in some applications. Stabilised finite element methods, on the other hand, do not satisfy this property, even when discontinuous pressures are used, due to the pressure jumps added to the formulation. One possibility is to propose a post-processing of the discrete velocity, by means of the lowest order Raviart-Thomas element, as it has been done, for instance, in [BV10] in shape-regular triangular meshes. Another possibility to address this issue is to add a grad-div stabilisation term, this is, a consistent term of the form $\gamma(\operatorname{div} \boldsymbol{u}, \operatorname{div} \boldsymbol{v})_{\Omega}$, where $\gamma>0$ is a stabilisation parameter. The introduction of this term was first proposed in [FH88] and it has been extensively used to improve the control of the discrete divergence for the Navier-Stokes equation and coupled problems (see, e.g., [LM+09, GL+12]). An analysis of this method can be found in [OR04], and, especially in [JJ+14], where a very detailed analysis and discussion on the selection of the stabilisation parameter is presented.

The objective of this paper is to propose and analyse a stabilised finite element method for the Oseen problem on anisotropic quadrilateral meshes containing corner patches. The stabilisation terms related to the pressure are those from the method given in [LS13], supplied with appropriate (selected) edge terms to make the method stable independently of the aspect ratio, and the presence of corner patches. Since the method from [LS13] is based on the refinement of a macro-element mesh, then the present approach also requires some level of structure of the meshes. Concerning the stabilisation mechanisms for the convection, the stabilising terms are based on the LPS stabilisation ones, augmented with a grad-div/LPS term (this is, a term penalising the fluctuations of the divergence, rather than the divergence itself, as it was done, e.g., in [BV10]). One extra feature of the method is that the velocity is locally mass-conservative, at least in the macro-elements, and this fact is further imposed by the grad-div stabilisation term. The analysis of the method follows the very general approach given in [MT15], supplied with the new proof of stability for the pressure, which generalises the one presented in [BW15].

This text is organised as follows. Section 2 first defines the Oseen problem and its weak formulation. Then, the assumptions associated to the mesh are given. After that, results for the Stokes problem are extended to the class of meshes considered in this work. In particular, we prove the existence of a subspace $G$ of the pressure space such that the pair $\boldsymbol{V}_{\mathcal{P}} \times G$ (where $\boldsymbol{V}_{\mathcal{P}}$ is the discrete velocity space used later on) satisfies a uniform LBB condition (this is, an inf-sup condition where the inf-sup constant does not depend on the aspect ratio). This is confirmed numerically. Additionally, the existence of a weakly divergence preserving interpolant is stated. In Section 3 we then give the general framework for the methods in this text. In Sections 4 and 5 stability and a priori estimates are derived. The definition and analysis of the methods leaves the choice of stabilisation terms and parameters flexible. Section 6 fixes the latter for the numerical experiments in Section 7. 


\section{Notation and preliminary results}

Throughout, constants with capital $C$ are independent of data, whereas constants with a lower case $c$ may depend on data. Both the instances of $C$ and $c$ are independent of all geometric properties of the mesh. We use standard notation for Sobolev spaces; for instance, for $\omega \subset \mathbb{R}^{2},|\cdot|_{1, \omega}$ and $\|\cdot\|_{0, \omega}$ denote the $H^{1}(\omega)$-seminorm and $L^{2}(\omega)$-norm, respectively, and $L_{0}^{2}(\omega)$ denotes the space of functions in $L^{2}(\omega)$ with zero mean in $\omega$. Furthermore, by $(v, w)_{\omega}$ we denote the inner product in $L^{2}(\omega)$. Vector-valued spaces are bold-faced, e.g. $\boldsymbol{H}_{0}^{1}(\omega)=\left[H_{0}^{1}(\omega)\right]^{2}$, but the same notation for norms and inner products is used.

\subsection{The problem of interest}

Let $\Omega \subset \mathbb{R}^{2}$ be a polygonal, bounded and connected domain. Then, given $\boldsymbol{f} \in \boldsymbol{L}^{2}(\Omega)$ we consider the following Oseen problem

$$
\begin{aligned}
-\nu \Delta \boldsymbol{u}+(\boldsymbol{b} \cdot \nabla) \boldsymbol{u}+\sigma \boldsymbol{u}+\nabla p & =\boldsymbol{f} & & \text { in } \Omega, \\
\operatorname{div} \boldsymbol{u} & =0 & & \text { in } \Omega, \\
\boldsymbol{u} & =0 & & \text { on } \partial \Omega,
\end{aligned}
$$

subject to $\langle p\rangle_{\Omega}=0$, where $\langle q\rangle_{\omega}$ denotes the meanvalue of $q$ over $\omega \subset \Omega$. For simplicity we suppose $\nu$ is a positive viscosity constant, $\sigma$ is a non-negative constant and $\boldsymbol{b} \in$ $\boldsymbol{H}(\operatorname{div}, \Omega) \cap \boldsymbol{L}^{\infty}(\Omega)$, with $\operatorname{div} \boldsymbol{b}=0$, is a given velocity field. The weak formulation of Problem (2.1) is given by:

Find $(\boldsymbol{u}, p) \in \boldsymbol{V} \times Q:=\boldsymbol{H}_{0}^{1}(\Omega) \times L_{0}^{2}(\Omega)$ such that

$$
\mathfrak{B}((\boldsymbol{u}, p),(\boldsymbol{v}, q))=(\boldsymbol{f}, \boldsymbol{v})_{\Omega} \quad \text { for all } \quad(\boldsymbol{v}, q) \in \boldsymbol{V} \times Q,
$$

where

$$
\begin{aligned}
\mathfrak{B}((\boldsymbol{u}, p),(\boldsymbol{v}, q)) & :=a(\boldsymbol{u}, \boldsymbol{v})-(\operatorname{div} \boldsymbol{v}, p)_{\Omega}-(\operatorname{div} \boldsymbol{u}, q)_{\Omega}, \\
a(\boldsymbol{u}, \boldsymbol{v}) & :=\nu(\nabla \boldsymbol{u}, \nabla \boldsymbol{v})_{\Omega}+((\boldsymbol{b} \cdot \nabla) \boldsymbol{u}, \boldsymbol{v})_{\Omega}+\sigma(\boldsymbol{u}, \boldsymbol{v})_{\Omega} .
\end{aligned}
$$

Using integration by parts and $\operatorname{div} \boldsymbol{b}=0$ the bilinear form $a$ induces the norm

$$
\|\boldsymbol{v}\|_{a}^{2}:=a(\boldsymbol{v}, \boldsymbol{v})=\nu|\boldsymbol{v}|_{1, \Omega}^{2}+\sigma\|\boldsymbol{v}\|_{0, \Omega}^{2} \quad \text { for all } \quad \boldsymbol{v} \in \boldsymbol{V} .
$$

If $\sigma=0$, then thanks to the Poincaré inequality

$$
\exists C_{\Omega}>0 \quad, \quad \forall \boldsymbol{v} \in \boldsymbol{V}:\|\boldsymbol{v}\|_{0, \Omega} \leq C_{\Omega}|\boldsymbol{v}|_{1, \Omega},
$$

$\|\cdot\|_{a}$ remains a norm. The following continuity estimates will be of use in the stability and convergence analysis.

Lemma 2.1. For all $\boldsymbol{w}, \boldsymbol{v} \in \boldsymbol{V}$ the following inequalities hold

$$
\|\boldsymbol{v}\|_{0, \Omega}^{2} \leq \frac{C_{\Omega}^{2}}{\nu+\sigma C_{\Omega}^{2}}\|\boldsymbol{v}\|_{a}^{2},
$$

and

$$
a(\boldsymbol{w}, \boldsymbol{v}) \leq c_{a}\|\boldsymbol{w}\|_{a}|\boldsymbol{v}|_{1, \Omega} \quad \text { where } \quad c_{a}:=\frac{\nu+\sigma C_{\Omega}^{2}+b_{\infty, \Omega} C_{\Omega}}{\left(\nu+\sigma C_{\Omega}^{2}\right)^{1 / 2}}
$$

with $C_{\Omega}$ from (2.6) and $b_{\infty, \omega}:=\|\boldsymbol{b}\|_{\infty, \omega}$ for $\omega \subseteq \bar{\Omega}$. 
Proof. Using the Poincaré inequality (2.6) we get

$$
\|\boldsymbol{v}\|_{a}^{2} \geq \frac{\nu}{C_{\Omega}^{2}}\|\boldsymbol{v}\|_{0, \Omega}^{2}+\sigma\|\boldsymbol{v}\|_{0, \Omega}^{2}=\frac{\nu+\sigma C_{\Omega}^{2}}{C_{\Omega}^{2}}\|\boldsymbol{v}\|_{0, \Omega}^{2},
$$

which proves (2.7). To prove (2.8), we consider (2.4), (2.5) and estimate term by term. First, we obtain

$$
\begin{aligned}
\nu(\nabla \boldsymbol{w}, \nabla \boldsymbol{v})_{\Omega}+\sigma(\boldsymbol{w}, \boldsymbol{v})_{\Omega} & \leq\left(\nu|\boldsymbol{v}|_{1, \Omega}^{2}+\sigma\|\boldsymbol{v}\|_{0, \Omega}^{2}\right)^{1 / 2}\|\boldsymbol{w}\|_{a} \\
& \leq\left(\nu+\sigma C_{\Omega}^{2}\right)^{1 / 2}|\boldsymbol{v}|_{1, \Omega}\|\boldsymbol{w}\|_{a} .
\end{aligned}
$$

Now, integrating by parts, using $\operatorname{div} \boldsymbol{b}=0$, and (2.7) we get

$$
\left|((\boldsymbol{b} \cdot \nabla) \boldsymbol{w}, \boldsymbol{v})_{\Omega}\right|=\left|((\boldsymbol{b} \cdot \nabla) \boldsymbol{v}, \boldsymbol{w})_{\Omega}\right| \leq b_{\infty, \Omega}|\boldsymbol{v}|_{1, \Omega}\|\boldsymbol{w}\|_{0, \Omega} \leq \frac{b_{\infty, \Omega} C_{\Omega}}{\left(\nu+\sigma C_{\Omega}^{2}\right)^{1 / 2}}|\boldsymbol{v}|_{1, \Omega}\|\boldsymbol{w}\|_{a} .
$$

Adding these last two estimates proves (2.8).

Finally, the inf-sup (or LBB) condition

$$
\inf _{q \in Q} \sup _{\boldsymbol{v} \in \boldsymbol{V}} \frac{(q, \operatorname{div} \boldsymbol{v})_{\Omega}}{|\boldsymbol{v}|_{1, \Omega}\|q\|_{0, \Omega}} \geq \beta_{\Omega}>0
$$

holds, see for instance [GR86, pp. 58-61]. With these last ingredients, and applying standard arguments in variational problems with constraints (see, e.g., [GR86]), we conclude that the Oseen problem (2.2) has a unique solution.

\subsection{Partitions and finite elements}

In order to construct partition $\mathcal{P}$ we start from an initial macro element partition $\mathcal{M}$ that consists of closed parallelograms and satisfies a maximal angle condition. We suppose that $\mathcal{M}$ is conforming, that is, the non-empty intersection of $M, M^{\prime} \in \mathcal{M}$ is either a single common point or a shared edge. It is worth mentioning that partition $\mathcal{M}$ is allowed to be highly anisotropic and contain corner patches, that is, a drastic change of stretching in two directions may occur in some parts of the mesh. See for example the areas around the shaded cells in Figures 1-3.

We define the partition $\mathcal{P}$ as a uniform refinement of $\mathcal{M}$, and state the main definitions and properties of $\mathcal{P}$ :

- Let $\mathcal{E}_{\mathcal{P}}$ denote the set of interior edges of $\mathcal{P}$. Throughout we use $M$ to denote an element of $\mathcal{M}$ and refer to it as macro element, and use $K$ to denote elements of $\mathcal{P}$. Additionally, we use $|\omega|$ to denote the area of $\omega \subset \mathbb{R}^{2}$ and $|e|$ to denote the length of an edge.

- The uniform refinement splits each macro element $M \in \mathcal{M}$ into $K_{1}, K_{2}, K_{3}, K_{4} \in \mathcal{P}$, such that $\left|K_{i}\right|=|M| / 4(i=1, . ., 4)$, see Figure 1 .

- For $M \in \mathcal{M}$, let $\mathcal{E}_{M} \subseteq \mathcal{E}_{\mathcal{P}}$ denote the set of its interior edges, dashed in Fig. 1-3. 
- The aspect ratio of a cell $K \in \mathcal{P}$ is defined by $\varrho_{K}:=\min _{e \subset \partial K}|K| /|e|^{2}$. The mesh aspect ratio is defined by $\varrho:=\min _{K \in \mathcal{P}} \varrho_{K}$.

- Let $\mathcal{C}$ be the set of corners, that is, nodes $\boldsymbol{c}$ of the mesh $\mathcal{M}$ towards which the mesh is refined, denoted by filled circles in Fig. 1-3. For $c \in \mathcal{C}$, we denote by $\omega_{\boldsymbol{c}}$ the area around $\boldsymbol{c}$ that is partitioned in a shape-regular way (shaded in Fig. 1-3). Moreover, for every $\boldsymbol{c} \in \mathcal{C}$, we select a single edge $\gamma_{\boldsymbol{c}} \in \mathcal{E}_{\mathcal{P}}$ that separates a small corner macro element (shaded) from a highly stretched neighbouring macro element, e.g., the embraced edges in Fig. 1-3. The selected edges $\gamma_{c}$ are collected in the set $\mathcal{E}_{\mathcal{C}}$.

Even if the above-stated hypotheses allow more general situations, from now on we will restrict our analysis to meshes of the type depicted in Figures 1-3. In particular, we will not consider the case of graded meshes or meshes in which the change between $\omega_{c}$ and the complement is more subtle than the one from these figures.
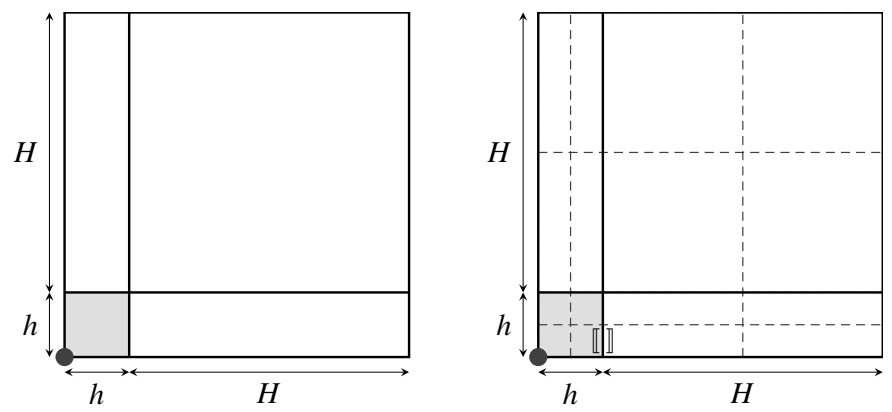

Fig. 1 Partition $\mathcal{M}$ (left) and $\mathcal{P}$ (right). We call this $\mathcal{M}$ corner patch.
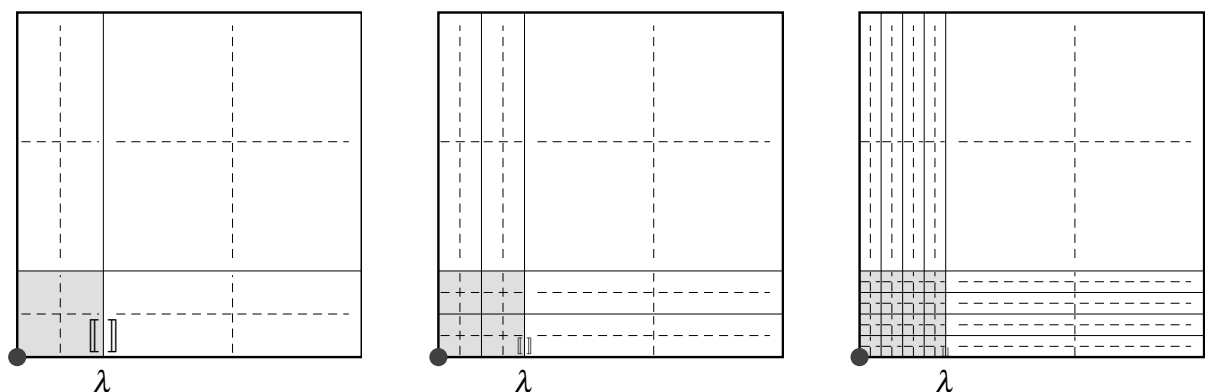

FIG. 2 Corner patches on $[0, \lambda+H]^{2}$ whose corners were refined $r$ times $(r=0,1,2)$.

It is worth mentioning that the condition "P arises from a uniform refinement of $\mathcal{M}$ " still allows local (macro-element based) refinements, as described in [LS13]. In particular, instead of $\mathcal{M}$, an initial partition $\mathcal{M}_{r}$, that contains corner patches that have been refined uniformly $r$-times, may be used as a macro-element mesh for $\mathcal{P}$, cf. Figure 2 , where the partitions $\mathcal{M}_{0}, \mathcal{M}_{1}, \mathcal{M}_{2}$ and $\mathcal{P}_{0}, \mathcal{P}_{1}, \mathcal{P}_{2}$ have been depicted. To lighten the notation, we remove the subindex $r$ whenever it is clear from the context, but we keep in mind that the partitions $\mathcal{M}$ and $\mathcal{P}$ have been refined, as in Fig. 2, $r$ times.

Finally, we define the finite element spaces

$$
\boldsymbol{V}_{\ell, \mathcal{P}}:=\left\{\boldsymbol{v} \in \boldsymbol{V}:\left.\boldsymbol{v}\right|_{K} \in \mathbb{Q}_{\ell}(K)^{2} \quad \text { for all } \quad K \in \mathcal{P}\right\}, \ell=1,2,
$$




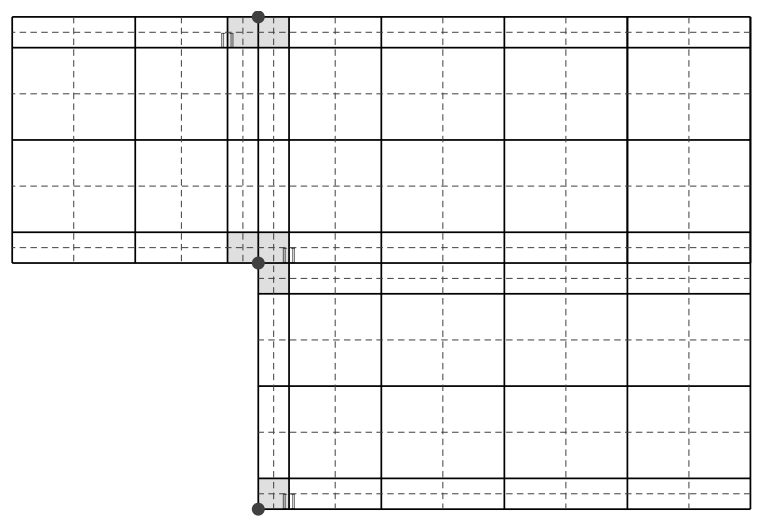

FIG. 3 An anisotropic mesh for flow over step.

and

$$
Q_{\mathcal{P}}:=\left\{q \in Q:\left.q\right|_{K} \in \mathbb{P}_{0}(K) \text { for all } K \in \mathcal{P}\right\},
$$

where, as usual, $\mathbb{Q}_{\ell}(K)$ denotes the space of polynomials of degree less that, or equal to, $\ell$ in each variable, and $\mathbb{P}_{0}(K)$ denotes the space of constant functions in $K$. We seek an approximation of the solution $(\boldsymbol{u}, p)$ of Problem (2.1) within the discrete space $\boldsymbol{V}_{1, \mathcal{P}} \times Q_{\mathcal{P}}$.

\subsection{Preliminary results}

It is a well known fact that $\boldsymbol{V}_{1, \mathcal{P}} \times Q_{\mathcal{P}}$ is not inf-sup stable, even on shape-regular meshes. On the other hand, since $\boldsymbol{V}_{1, \mathcal{P}}$ and $\boldsymbol{V}_{2, \mathcal{M}}$ share the same degrees of freedom, $\boldsymbol{V}_{1, \mathcal{P}} \times Q_{\mathcal{M}}$ is inf-sup stable. Now, since $\mathcal{M}$ contains corner patches, then the inf-sup constant of the latter pair is affected by the aspect ratio of $\mathcal{M}$. More precisely, applying the results from [AC00] (see also [ABW15]) we conclude that

$$
\inf _{q \in Q_{\mathcal{M}}} \sup _{\boldsymbol{v} \in \boldsymbol{V}_{1, \mathcal{P}}} \frac{(q, \operatorname{div} \boldsymbol{v})_{\Omega}}{|\boldsymbol{v}|_{1, \Omega}\|q\|_{0, \Omega}}=\beta_{\mathcal{M}} \geq C \sqrt{\varrho}
$$

and this bound is sharp. This issue is then solved in the next result where we impose a minimal set of additional constraints to obtain a uniformly inf-sup stable subspace $G$ of $Q_{\mathcal{M}}$. In this lemma, and thereafter, for a function $q$, $\llbracket q \rrbracket_{\gamma}$ will denote its jump across the edge $\gamma$.

Lemma 2.2. Let $G \subset Q_{\mathcal{M}} \subset Q_{\mathcal{P}}$ be the space defined by

$$
G:=\left\{q \in Q_{\mathcal{M}}: \llbracket q \rrbracket_{\gamma_{c}}=0 \quad \text { for every } \quad \gamma_{\boldsymbol{c}} \in \mathcal{E}_{\mathcal{C}}\right\} .
$$

Then, the following inf-sup condition holds

$$
\sup _{\boldsymbol{v} \in \boldsymbol{V}_{1, \mathcal{P}}} \frac{(\operatorname{div} \boldsymbol{v}, q)_{\Omega}}{|\boldsymbol{v}|_{1, \Omega}} \geq \beta_{G}\|q\|_{0, \Omega} \quad \text { for all } \quad q \in G,
$$

with a constant $\beta_{G} \geq \max \left\{\beta_{\mathcal{M}}, C / 2^{r}\right\}$, where $C$ is independent of the mesh, data of the problem, and $r$ (the number of times the initial macro-element mesh has been refined, 
see Figure 2). Equivalently, the following inf-sup deficiency holds

$$
\sup _{\boldsymbol{v} \in \boldsymbol{V}_{1, \mathcal{P}}} \frac{(\operatorname{div} \boldsymbol{v}, q)_{\Omega}}{|\boldsymbol{v}|_{1, \Omega}} \geq \beta_{G}\left\|\Pi_{G} q\right\|_{0, \Omega}-\left\|q-\Pi_{G} q\right\|_{0, \Omega} \quad \text { for all } \quad q \in Q_{\mathcal{P}}
$$

where $\Pi_{G}: Q_{\mathcal{P}} \rightarrow G$ stands for the $L^{2}(\Omega)$-projection onto $G$.

Proof. The proof follows a similar path as in [ABW15] allowing the extension to refined corner patches. For completeness we include an abridged version here. We first prove (2.15). Since $G \subset Q_{\mathcal{M}}$, we have $\beta_{G} \geq \beta_{\mathcal{M}}$. For the alternative $\beta_{G} \geq C / 2^{r}$, let us define

$$
Q_{\mathcal{M}}^{*}:=\left\{q \in Q_{\mathcal{M}}:\langle q\rangle_{\omega_{c}}=0 \text { for } c \in \mathcal{C}\right\} .
$$

Let now $q^{*} \in Q_{\mathcal{M}}^{*}$. As in [ABW15, Corollary 3.1] there exists $\boldsymbol{v}^{*} \in \boldsymbol{V}_{1, \mathcal{P}}$ such that $\left.\boldsymbol{v}^{*}\right|_{\omega_{\boldsymbol{c}}} \in \boldsymbol{H}_{0}^{1}\left(\omega_{\boldsymbol{c}}\right)$ for every $\boldsymbol{c} \in \mathcal{C}$, and

$$
\left(\operatorname{div} \boldsymbol{v}^{*}, q^{*}\right)_{\Omega}=\left\|q^{*}\right\|_{0, \Omega}^{2} \quad \text { and } \quad\left|\boldsymbol{v}^{*}\right|_{1, \Omega} \leq \hat{C}\left\|q^{*}\right\|_{0, \Omega}
$$

where $\hat{C}>0$ depends only on $\Omega$. In particular, this constant $\hat{C}$ is independent of $r$. Next, we decompose $q \in G$ into $q=\Pi_{\mathcal{C}} q+q^{*}$ where $\left.\Pi_{\mathcal{C}} q\right|_{\omega_{\boldsymbol{c}}}($ for $\boldsymbol{c} \in \mathcal{C})$ and $\left.\Pi_{\mathcal{C}} q\right|_{\Omega \backslash\left(\cup_{\left.\boldsymbol{c} \in \mathcal{C} \omega_{\boldsymbol{c}}\right)}\right.}$ are constants, and $q^{*} \in Q_{\mathcal{M}}^{*}$. Then, since $\left(\operatorname{div} \boldsymbol{v}^{*}, \Pi_{\mathcal{C}} q\right)_{\Omega}=0$ we get $\left(\operatorname{div} \boldsymbol{v}^{*}, q\right)=\left\|q^{*}\right\|_{0, \Omega}^{2}$. Therefore, (2.17) implies (2.15), once the following is proved

$$
\|q\|_{0, \Omega} \leq C 2^{r}\left\|q^{*}\right\|_{0, \Omega} .
$$

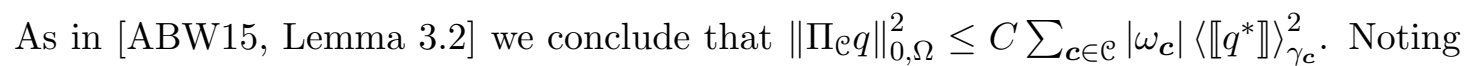
that $\gamma_{\boldsymbol{c}} \subset M_{\boldsymbol{c}} \cap M_{\boldsymbol{c}}^{\prime}$, with $M_{\boldsymbol{c}}, M_{\boldsymbol{c}}^{\prime} \in \mathcal{M},\left|M_{c}\right| \leq\left|M_{\boldsymbol{c}}^{\prime}\right|$, and using that $\left|\omega_{\boldsymbol{c}}\right|\left|M_{\boldsymbol{c}}\right|^{-1}=2^{2 r}$, each of these jumps is bounded as

$$
\left|\omega_{\boldsymbol{c}}\right|\left\langle\llbracket q^{*} \rrbracket\right\rangle_{\gamma_{\boldsymbol{c}}}^{2} \leq C\left|\omega_{\boldsymbol{c}}\right|\left|M_{\boldsymbol{c}}\right|^{-1}\left\|q^{*}\right\|_{0, M_{c} \cup M_{c}^{\prime}}^{2} \leq C 2^{2 r}\left\|q^{*}\right\|_{0, \Omega}^{2},
$$

and then (2.18) follows.

Finally, given (2.15), the proof of [ABW15, Lemma 4.1] implies (2.16). The reverse follows using only $\Pi_{G} q=q$ for $q \in G$. This finishes the proof.

Remark 2.3. We stress the fact that $\beta_{G}$ only depends on how refined the partition $\mathcal{M}$ is. This is reflected by the factor $2^{r}$ in $\beta_{G}$. This unfortunate behaviour can be solved easily by limiting the number of refinements and instead moving $\lambda$ closer to the nodes $\boldsymbol{c}$, since $\beta_{G}$ is bounded below by a constant independent of $\lambda$.

The next result appears as a natural consequence of the previous Lemma, and standard finite element approximation results for variational problems with constraints. In particular, it states that an approximation, denoted $\boldsymbol{u}_{I}$, of $\boldsymbol{u}$ can be built in such a way that it is weakly divergence-free (in the macro-elements) and has optimal approximation properties. This approximation somehow generalises the divergence-preserving interpolation operator from [BLR12] to a different class of meshes, at the cost of providing only a global approximation result. 
Lemma 2.4. Let $G \subset Q_{\mathcal{P}}$ be defined as in Lemma 2.2. Then, there exists $\boldsymbol{u}_{I} \in \boldsymbol{V}_{1, \mathcal{P}}$ such that

$$
\left(\operatorname{div}\left(\boldsymbol{u}-\boldsymbol{u}_{I}\right), q\right)_{\Omega}=0 \quad \text { for all } q \in G,
$$

and

$$
\left|\boldsymbol{u}-\boldsymbol{u}_{I}\right|_{1, \Omega} \leq 2\left(1+\beta_{G}^{-1}\right) \inf _{\boldsymbol{v}_{\mathcal{P}} \in \boldsymbol{V}_{1, \mathcal{P}}}\left|\boldsymbol{u}-\boldsymbol{v}_{\mathcal{P}}\right|_{1, \Omega} .
$$

Proof. Let $\left(\phi_{\mathcal{P}}, \chi_{\mathcal{P}}\right) \in \boldsymbol{V}_{1, \mathcal{P}} \times G$ be the solution of the following auxiliary problem:

$$
\begin{aligned}
\left(\nabla \phi_{\mathcal{P}}, \nabla \boldsymbol{v}\right)_{\Omega}- & \left(\operatorname{div} \boldsymbol{v}, \chi_{\mathcal{P}}\right)_{\Omega}=(\nabla \boldsymbol{u}, \nabla \boldsymbol{v})_{\Omega} & & \text { for all } \boldsymbol{v} \in \boldsymbol{V}_{1, \mathcal{P}} \\
& \left(\operatorname{div} \phi_{\mathcal{P}}, q\right)_{\Omega}=(\operatorname{div} \boldsymbol{u}, q)_{\Omega} & & \text { for all } q \in G .
\end{aligned}
$$

The well-posedness of this problem is a consequence of (2.15). Then, defining $\boldsymbol{u}_{I}:=\phi_{\mathcal{P}}$, (2.19) follows immediately from (2.21). Moreover, since $\left(\boldsymbol{u}_{I}, \chi_{\mathcal{P}}\right)$ is a finite element approximation of $(\boldsymbol{u}, 0),(2.20)$ follows by standard arguments, see e.g. [GR86, p.115], or [Joh17, Lemma 3.60 and Theorem 4.21].

Remark 2.5. We finish this section by providing some more insight in the behavior of the inf-sup constant $\beta_{\mathcal{M}}$ as the corner patches get refined. Our aim is to show that this constant, not only doesn't degenerate, but it actually increases with $r$. We remind that we have dropped the subscript $r$ whenever it is clear from the context, but we keep in mind that the macro-element patch $\mathcal{M}$ has been refined $r$ times.

First, from (2.13) and (2.17) we conclude that the spurious mode on the (refined) corner patch in Figure 2 is given by the function connecting the (uniformly stable) average free spaces on $\omega_{\boldsymbol{c}}:=[0, \lambda] \times[0, \lambda]$ and $\Omega \backslash \omega_{\boldsymbol{c}}$, i.e.

$$
q_{B}:=\chi_{\omega_{c}}-\frac{\left|\omega_{c}\right|}{\left|\Omega \backslash \omega_{c}\right|} \chi_{\Omega \backslash \omega_{c}} .
$$

Let us first define the quantity

$$
\tilde{\beta}_{r}:=\left\|q_{B}\right\|_{0, \Omega}^{-1} \sup _{\boldsymbol{v} \in \boldsymbol{V}_{1, \mathcal{P}}} \frac{\left(q_{B}, \operatorname{div} \boldsymbol{v}\right)_{\Omega}}{|\boldsymbol{v}|_{1, \Omega}} .
$$

Next, using (2.17) and the definition of the space $Q_{\mathcal{M}}^{*}$ we can easily see that

$$
\sup _{\boldsymbol{v} \in \boldsymbol{V}_{1, \mathcal{P}}} \frac{(\operatorname{div} \boldsymbol{v}, q)_{\Omega}}{|\boldsymbol{v}|_{1, \Omega}} \geq \frac{1}{\hat{C}}\left\|q-\frac{\left(q, q_{B}\right)_{\Omega}}{\left\|q_{B}\right\|_{0, \Omega}^{2}} q_{B}\right\|_{0, \Omega},
$$

for all $q \in Q_{\mathcal{P}}$. Then, using [Joh17, Theorem 3.89], we conclude that

$$
\beta_{\mathcal{M}}=\frac{\hat{C}^{-1}}{1+\hat{C}^{-1}+\tilde{\beta}_{r}} \tilde{\beta}_{r}
$$

Thus, $\beta_{\mathcal{M}}$ is an increasing function of $\tilde{\beta}_{r}$. Moreover, since the space $\boldsymbol{V}_{1, \mathcal{P}}$ becomes richer as $r$ increases, then $\tilde{\beta}_{r}$ increases with $r$. Thus, we have $\beta_{\mathcal{M}_{0}} \leq \beta_{\mathcal{M}_{1}} \leq \ldots \leq \beta_{\mathcal{M}_{r}} \leq C \sqrt{\rho_{0}}$, where $\rho_{0}$ is the aspect ratio of the initial macro-element partition $\mathcal{M}_{0}$. In Table 1 below we confirm this claim numerically. 


\subsection{Numerical confirmation (part 1)}

In this section we show the improvement of $\beta_{G}$ over $\beta_{\mathcal{M}}$. For simplicity we restrict the presentation of $\beta_{G}$ to partitions on the unit square $\Omega=(0,1) \times(0,1)$. To this end, we define a parametrized (by $\lambda>0$ ), refined corner patch $\mathcal{M}_{r}$ as the tensor-product of the following one-dimensional interval subdivision of $[0,1]$. The parameter $\lambda<1 / 2$ separates a coarse and a fine region in $[0,1]$. The interval $[0, \lambda]$ is split into $2^{r}$ intervals of length $\lambda / 2^{r}$ and $[\lambda, 1]$ remains unsplit. Figure 2 shows these macro-element meshes $\mathcal{M}_{r}$ for $r=0,1,2$ as continuous lines. The subspace $G \subset Q_{\mathcal{M}}$ additionally imposes the continuity across the edges in $\mathcal{E}_{\mathcal{C}}$ for each case.

We have computed $\beta_{G}$ and $\beta_{\mathcal{M}}$ for different levels of refinements while letting $\lambda \rightarrow 0$. The results are depicted in Figure 4 . The constants $\beta_{G}$ remain bounded below by a constant independent of $\lambda$, as predicted by Lemma 2.2. Moreover, to confirm the claim made in Remark 2.5 we have computed the constant $\beta_{\mathcal{M}}$ for different values of $r$ and different values of $\lambda$. We report the obtained values in Table 1 where it can be seen that the value of the inf-sup constant $\beta_{\mathcal{M}}$ grows with $r$.
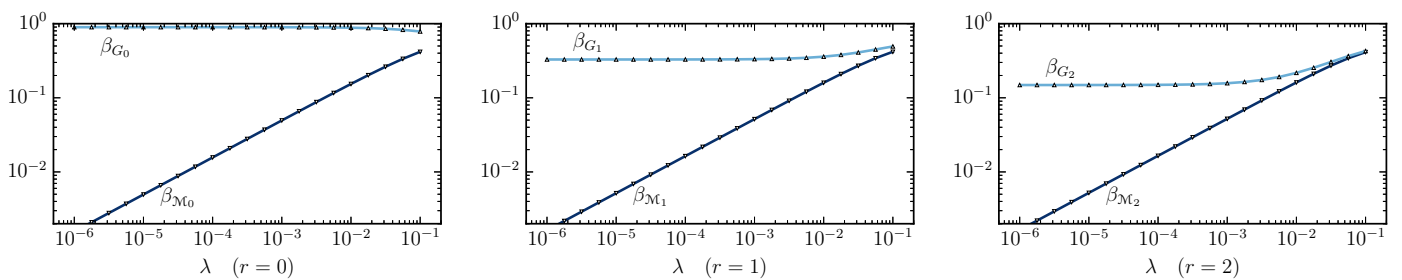

FIG. 4 Constants $\beta_{G}$ and $\beta_{\mathcal{M}}$, for $r=0,1,2$.

TABLE 1. A numerical confirmation of Remark 2.5

\begin{tabular}{lcccc}
\hline$\lambda$ & $\beta_{\mathcal{M}_{0}}$ & $\beta_{\mathcal{M}_{1}}$ & $\beta_{\mathcal{M}_{2}}$ & $\beta_{\mathcal{M}_{3}}$ \\
\hline $10^{-3}$ & $4.947 \cdot 10^{-2}$ & $5.157 \cdot 10^{-2}$ & $5.207 \cdot 10^{-2}$ & $5.220 \cdot 10^{-2}$ \\
$10^{-4}$ & $1.567 \cdot 10^{-2}$ & $1.634 \cdot 10^{-2}$ & $1.650 \cdot 10^{-2}$ & $1.654 \cdot 10^{-2}$ \\
$10^{-5}$ & $4.957 \cdot 10^{-3}$ & $5.169 \cdot 10^{-3}$ & $5.220 \cdot 10^{-3}$ & $5.233 \cdot 10^{-3}$ \\
\hline
\end{tabular}

\section{The stabilised method for the Oseen equation}

The stabilised method proposed in this work reads: Find $\left(\boldsymbol{u}_{\mathcal{P}}, p_{\mathcal{P}}\right) \in \boldsymbol{V}_{1, \mathcal{P}} \times Q_{\mathcal{P}}$ such that

$$
\mathfrak{B}_{s}\left(\left(\boldsymbol{u}_{\mathcal{P}}, p_{\mathcal{P}}\right),\left(\boldsymbol{v}_{\mathcal{P}}, q_{\mathcal{P}}\right)\right)=\left(\boldsymbol{f}, \boldsymbol{v}_{\mathcal{P}}\right)_{\Omega} \quad \text { for all } \quad\left(\boldsymbol{v}_{\mathcal{P}}, q_{\mathcal{P}}\right) \in \boldsymbol{V}_{1, \mathcal{P}} \times Q_{\mathcal{P}},
$$

where

$$
\mathfrak{B}_{s}((\boldsymbol{u}, p),(\boldsymbol{v}, q)):=\mathfrak{B}((\boldsymbol{u}, p),(\boldsymbol{v}, q))+s_{v}(\boldsymbol{u}, \boldsymbol{v})-s_{p}(p, q),
$$

and $s_{v}$ and $s_{p}$ are symmetric, positive semi-definite bilinear forms aimed at stabilising velocity and pressure, respectively. In order to prove stability and a priori estimates we need to make assumptions on $s_{v}$ and $s_{p}$. For this purpose, we define

$$
|\boldsymbol{v}|_{s_{v}}^{2}:=s_{v}(\boldsymbol{v}, \boldsymbol{v}) \quad \text { and } \quad\|\boldsymbol{v}\|_{a+s}^{2}:=\|\boldsymbol{v}\|_{a}^{2}+|\boldsymbol{v}|_{s_{v}}^{2}
$$


and the bilinear form

$$
s_{v}^{\operatorname{div}}(\boldsymbol{u}, \boldsymbol{v}):=\sum_{K \in \mathcal{P}} \gamma_{K}\left(\kappa_{K}(\operatorname{div} \boldsymbol{u}), \kappa_{K}(\operatorname{div} \boldsymbol{v})\right)_{K}, \quad \gamma_{K} \geq 0,
$$

where $\kappa_{\omega}:=\mathrm{id}-\langle\cdot\rangle_{\omega}$ denotes the fluctuation operator. We now state the main assumptions on $s_{v}$ and $s_{p}$.

Assumption 3.1. Let $\boldsymbol{v}, \boldsymbol{w} \in \boldsymbol{V}$. There exists a positive constant $c_{s}$, which may depend on the data, but is independent of the mesh, such that

$$
s_{v}(\boldsymbol{w}, \boldsymbol{v}) \leq c_{s}|\boldsymbol{w}|_{s_{v}}|\boldsymbol{v}|_{1, \Omega} .
$$

Furthermore, $s_{v}$ is assumed to satisfy

$$
s_{v}^{d i v}(\boldsymbol{v}, \boldsymbol{v}) \leq s_{v}(\boldsymbol{v}, \boldsymbol{v}),
$$

where $s_{v}^{\text {div }}$ is given by the LPS-like term (3.4).

We remark that, thanks to the above hypotheses then $s_{v}(\cdot, \cdot)$ satisfies the following Cauchy-Schwarz inequality

$$
s_{v}(\boldsymbol{w}, \boldsymbol{v}) \leq s_{v}(\boldsymbol{w}, \boldsymbol{w})^{1 / 2} s_{v}(\boldsymbol{v}, \boldsymbol{v})^{1 / 2} .
$$

With the above constants we define

$$
\alpha:=\frac{1}{c_{a}^{2}+c_{s}^{2}},
$$

with $c_{a}$ and $c_{s}$ from (2.8) and (3.5), respectively. Then, the pressure stabilisation term is given by

$$
s_{p}(p, q):=\frac{\alpha_{p}}{4} \sum_{M \in \mathcal{M}} S_{M}(p, q)+\frac{\alpha_{p}}{4} \sum_{\gamma_{c} \in \mathcal{E}_{\mathrm{e}}} S_{\gamma_{c}}(p, q),
$$

with $\alpha_{p} \geq \alpha$, and

$$
\begin{aligned}
S_{M}(p, q) & :=\sum_{e \in \mathcal{E}_{M}} \frac{|M|}{4|e|}(\llbracket p \rrbracket, \llbracket q \rrbracket)_{e}, \\
S_{\gamma_{c}}(p, q) & :=\frac{\min \left\{|K|,\left|K^{\prime}\right|\right\}}{\left|\gamma_{c}\right|}(\llbracket p \rrbracket, \llbracket q \rrbracket)_{\gamma_{c}},
\end{aligned}
$$

where $K, K^{\prime} \in \mathcal{P}$ are such that $\gamma_{c}=K \cap K^{\prime}$.

Remark 3.2. For $q \in G$ we realise that $s_{p}(q, q)=0$. Consequently, $s_{p}$ only acts in the complement of $G$. Then, this scheme falls in the category of "minimal" stabilised methods, as described in the introduction. Moreover, if the (macro-element) mesh $\mathcal{M}$ does not contain corner patches, then $S_{\gamma_{c}}:=0$ and the present term $s_{p}$ appears as an extension of the one from [LS13] to the Oseen equation. 


\section{Stability of the method}

This section is devoted to proving that Method (3.1) is stable with a stability constant depending only on $\beta_{G}$. The norm that will be used is given by

$$
\|(\boldsymbol{v}, q)\|^{2}:=\|\boldsymbol{v}\|_{a+s}^{2}+\alpha\|q\|_{0, \Omega}^{2}+s_{p}(q, q) .
$$

The next result is the first step towards stability.

Lemma 4.1. Let $q_{\mathcal{P}} \in Q_{\mathcal{P}}, p \in H^{1}(\Omega)$ and $\Pi_{G}$ be the projection from Lemma 2.2. Then, the following holds

$$
\begin{aligned}
& \frac{1}{34} \alpha_{p}\left\|q_{\mathcal{P}}-\Pi_{G} q_{\mathcal{P}}\right\|_{0, \Omega}^{2} \leq s_{p}\left(q_{\mathcal{P}}, q_{\mathcal{P}}\right), \\
& s_{p}\left(q_{\mathcal{P}}, q_{\mathcal{P}}\right) \leq C \alpha_{p} \sum_{K \in \mathcal{P}}\left(\left\|p-q_{\mathcal{P}}\right\|_{0, K}^{2}+\left|e_{1, K}\right|^{2}\left\|\partial_{t_{1}} p\right\|_{0, K}^{2}+\left|e_{2, K}\right|^{2}\left\|\partial_{t_{2}} p\right\|_{0, K}^{2}\right),
\end{aligned}
$$

where $e_{1, K}$ and $e_{2, K}$ are two non-parallel edges of $K, \partial_{t_{i}}(i=1,2)$ are partial derivatives in their directions, and $C$ is a constant independent of mesh, angles, and data.

Proof. We start with (4.2). This proof uses notation and conventions from Figure 5. Our assumptions on the partitions $\mathcal{P}$ and $\mathcal{M}$ imply that every selected edge $\gamma_{\boldsymbol{c}} \in \mathcal{E}_{\mathcal{E}}$ (the embraced edge in Figure 5-right) satisfies $\gamma_{\boldsymbol{c}} \subset M \cap M^{\prime}$ where $M, M^{\prime} \in \mathcal{M}$ and $|M| \leq\left|M^{\prime}\right|$. For readability we define $\omega_{\gamma_{c}}:=M \cup M^{\prime}$. Now, from its definition $\Pi_{G} q$ is given by

$$
\left.\Pi_{G} q\right|_{M}= \begin{cases}\langle q\rangle_{\omega_{\gamma_{c}}} & \text { if } M \subset \omega_{\gamma_{c}}, \\ \langle q\rangle_{M} & \text { otherwise. }\end{cases}
$$

Therefore, bound (4.2) follows once we prove the local bounds

$$
\begin{aligned}
2 \alpha_{p}\left\|q_{\mathcal{P}}-\left\langle q_{\mathcal{P}}\right\rangle_{M}\right\|_{0, M}^{2} & \leq \alpha_{p} S_{M}\left(q_{\mathcal{P}}, q_{\mathcal{P}}\right), \\
\frac{2}{17} \alpha_{p}\left\|q_{\mathcal{P}}-\left\langle q_{\mathcal{P}}\right\rangle_{\omega_{\gamma_{c}}}\right\|_{\omega_{\gamma_{c}}}^{2} & \leq \alpha_{p}\left(S_{M}+S_{M^{\prime}}+S_{\gamma_{c}}\right)\left(q_{\mathcal{P}}, q_{\mathcal{P}}\right) .
\end{aligned}
$$

The first estimate has been proven as part of [LS13, Lemma 3.2]. We include here a different proof which supplies us with notation and arguments for $(4.5 \mathrm{~b})$. Let $M \in \mathcal{M}$ be a macro element such that $M \not \subset \omega_{\gamma_{c}}, \gamma_{c} \in \mathcal{E}_{\mathcal{C}}$. Since all cells $K \subset M$ have the same area, an orthogonal basis of $Q_{\mathcal{P}} \cap L_{0}^{2}(M)$ is given by (cf. Figure 5 , left)

$$
\begin{aligned}
\phi_{1, M} & :=\chi_{K_{1}}-\chi_{K_{2}}, \\
\phi_{2, M} & :=\chi_{K_{1} \cup K_{2}}-\chi_{K_{3} \cup K_{4}}, \\
\phi_{3, M} & :=\chi_{K_{3}}-\chi_{K_{4}},
\end{aligned}
$$
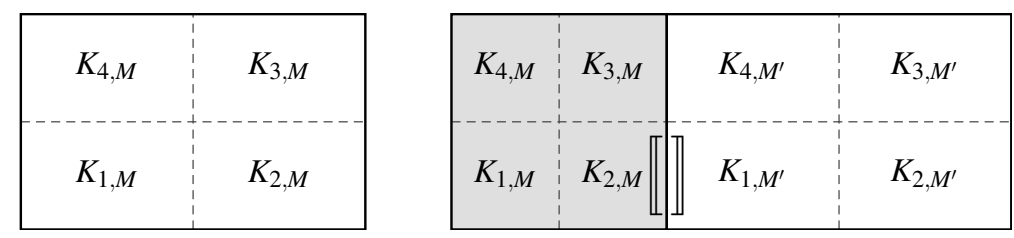

FIG. 5 A macro element $M \in \mathcal{M}$ (left) and set $\omega_{\gamma_{c}}$ (right) with cells $K_{i, M} \in \mathcal{P}$. 
where $\chi_{\omega}$ is the characteristic function of $\omega$. Below, we omit the subscript $M$ when it is clear from the context.

Let $r_{a}:=\left.\left(q_{\mathcal{P}}-\left\langle q_{\mathcal{P}}\right\rangle_{M}\right)\right|_{M} \in Q_{\mathcal{P}} \cap L_{0}^{2}(M)$. Then, $r_{a}=\sum_{i=1}^{3} \alpha_{i} \phi_{i}$ with appropriate coefficients $\alpha_{i}$, and using $\left|K_{i}\right|=|M| / 4,(i=1, \ldots, 4)$, the definition of $S_{M}, \llbracket r_{a} \rrbracket_{e} \in \mathbb{P}_{0}(e)$, and orthogonality of the basis we get

$$
\begin{aligned}
S_{M}\left(q_{\mathcal{P}}, q_{\mathcal{P}}\right) & =S_{M}\left(r_{a}, r_{a}\right)=\frac{|M|}{4} \sum_{e \in \mathcal{E}_{M}} \frac{1}{|e|}\left\|\llbracket r_{a} \rrbracket\right\|_{0, e}^{2}=\frac{|M|}{4} \sum_{e \in \mathcal{E}_{M}} \llbracket r_{a} \rrbracket_{e}^{2} \\
& =\frac{|M|}{4}\left[\left(2 \alpha_{1}\right)^{2}+\left(2 \alpha_{2}-\alpha_{1}-\alpha_{3}\right)^{2}+\left(2 \alpha_{3}\right)^{2}+\left(-2 \alpha_{2}-\alpha_{3}-\alpha_{1}\right)^{2}\right] \\
& =\frac{|M|}{4}\left[4 \alpha_{1}^{2}+4 \alpha_{3}^{2}+8 \alpha_{2}^{2}+2\left(\alpha_{1}+\alpha_{3}\right)^{2}\right] \\
& =2\left\|\alpha_{1} \phi_{1}\right\|_{0, M}^{2}+2\left\|\alpha_{3} \phi_{3}\right\|_{0, M}^{2}+2\left\|\alpha_{2} \phi_{2}\right\|_{0, M}^{2}+\frac{|M|}{2}\left(\alpha_{1}+\alpha_{3}\right)^{2} \\
& =2\left\|r_{a}\right\|_{0, M}^{2}+\frac{|M|}{2}\left(\alpha_{1}+\alpha_{3}\right)^{2},
\end{aligned}
$$

which proves $(4.5 \mathrm{a})$.

To prove (4.5b), we fix an edge $\gamma_{c} \in \mathcal{E}_{\mathcal{C}}$ and let $r_{b}:=\left.\left(q_{\mathcal{P}}-\left\langle q_{\mathcal{P}}\right\rangle_{\omega_{\gamma_{c}}}\right)\right|_{\omega_{\gamma_{c}}}$. Then

$$
r_{b}=\alpha_{0} \phi_{0}+r_{a}+r_{a}^{\prime}
$$

where $\phi_{0}=|M|^{-1} \chi_{M}-\left|M^{\prime}\right|^{-1} \chi_{M^{\prime}}, r_{a}=\sum_{i=1}^{3} \alpha_{i} \phi_{i, M}$ and $r_{a}^{\prime}=\sum_{i=1}^{3} \alpha_{i}^{\prime} \phi_{i, M^{\prime}}$. Using (4.7), the definition of $\phi_{0}$ and $|K| \leq\left|K^{\prime}\right|$ (since $|M| \leq\left|M^{\prime}\right|$ ) we get

$$
\left(S_{M}+S_{M^{\prime}}+S_{\gamma_{c}}\right)\left(q_{\mathcal{P}}, q_{\mathcal{P}}\right) \geq 2\left\|r_{a}\right\|_{0, M}^{2}+2\left\|r_{a}^{\prime}\right\|_{0, M^{\prime}}^{2}+\frac{|K|}{\left|\gamma_{c}\right|}\left\|\llbracket r_{b} \rrbracket\right\|_{0, \gamma_{c}}^{2} .
$$

It only remains to bound the last term. Using $\llbracket r_{b} \rrbracket_{\gamma_{c}}, \llbracket \phi_{0} \rrbracket_{\gamma_{c}} \in \mathbb{P}_{0}\left(\gamma_{c}\right)$ and the linearity of the jump, followed by $2 a b \leq \frac{1}{2} a^{2}+2 b^{2}$ we obtain

$$
\begin{aligned}
\frac{\left\|\llbracket r_{b} \rrbracket\right\|_{0, \gamma_{c}}^{2}}{\left|\gamma_{\boldsymbol{c}}\right|} & =\left(\llbracket \alpha_{0} \phi_{0} \rrbracket_{\gamma_{c}}+\alpha_{2}-\alpha_{2}^{\prime}-\alpha_{1}-\alpha_{1}^{\prime}\right)^{2} \\
& =\llbracket \alpha_{0} \phi_{0} \rrbracket_{\gamma_{c}}^{2}+2 \llbracket \alpha_{0} \phi_{0} \rrbracket_{\gamma_{c}}\left(\alpha_{2}-\alpha_{2}^{\prime}-\alpha_{1}-\alpha_{1}^{\prime}\right)+\left(\alpha_{2}-\alpha_{2}^{\prime}-\alpha_{1}-\alpha_{1}^{\prime}\right)^{2} \\
& \geq \frac{1}{2} \llbracket \alpha_{0} \phi_{0} \rrbracket_{\gamma_{c}}^{2}-\left(\alpha_{2}-\alpha_{2}^{\prime}-\alpha_{1}-\alpha_{1}^{\prime}\right)^{2} \\
& \geq \frac{1}{2} \llbracket \alpha_{0} \phi_{0} \rrbracket_{\gamma_{c}}^{2}-4\left(\alpha_{2}^{2}+\alpha_{2}^{\prime 2}+\alpha_{1}^{2}+\alpha_{1}^{\prime 2}\right),
\end{aligned}
$$

and conclude with $\varepsilon<1$ and $|K|=|M| / 4$

$$
\frac{|K|}{\left|\gamma_{\boldsymbol{c}}\right|}\left\|\llbracket r_{b} \rrbracket\right\|_{0, \gamma_{c}}^{2} \geq \varepsilon \frac{|K|}{\left|\gamma_{\boldsymbol{c}}\right|}\left\|\llbracket r_{b} \rrbracket\right\|_{0, \gamma_{c}}^{2} \geq \frac{\varepsilon}{8}|M| \llbracket \alpha_{0} \phi_{0} \rrbracket_{\gamma_{c}}^{2}-\varepsilon|M|\left(\alpha_{2}^{2}+\alpha_{2}^{\prime 2}+\alpha_{1}^{2}+\alpha_{1}^{\prime 2}\right) .
$$

Now, using the definition of $\phi_{0}$ and $|M| \leq\left|M^{\prime}\right|$ we get

$$
|M| \llbracket \alpha_{0} \phi_{0} \rrbracket_{\gamma_{c}}^{2}=\alpha_{0}^{2}|M|\left(\frac{1}{|M|}+\frac{1}{\left|M^{\prime}\right|}\right)^{2} \geq \alpha_{0}^{2}\left(\frac{1}{|M|}+\frac{1}{\left|M^{\prime}\right|}\right)=\left\|\alpha_{0} \phi_{0}\right\|_{0, \omega_{\gamma_{c}}}^{2},
$$


and

$$
\begin{aligned}
|M|\left(\alpha_{1}^{2}+\alpha_{1}^{\prime 2}+\alpha_{2}^{2}+\alpha_{2}^{\prime 2}\right) & \leq|M|\left(\alpha_{1}^{2}+\alpha_{2}^{2}\right)+\left|M^{\prime}\right|\left(\alpha_{1}^{\prime 2}+\alpha_{2}^{\prime 2}\right) \\
& \leq 2\left(\left\|r_{a}\right\|_{0, M}^{2}+\left\|r_{a}^{\prime}\right\|_{0, M^{\prime}}^{2}\right)
\end{aligned}
$$

Choosing $\varepsilon:=\frac{16}{17}$, inserting (4.9)-(4.11) into (4.8) and using that $\phi_{0}$ is orthogonal to $\phi_{i, M}, \phi_{i, M^{\prime}}, i=1,2,3$ leads to

$$
\left(S_{M}+S_{M^{\prime}}+S_{\gamma_{c}}\right)\left(q_{\mathcal{P}}, q_{\mathcal{P}}\right) \geq \frac{2}{17}\left(\left\|r_{a}\right\|_{0, M}^{2}+\left\|r_{a}^{\prime}\right\|_{0, M^{\prime}}^{2}+\left\|\alpha_{0} \phi_{0}\right\|_{0, \omega_{\gamma_{c}}}^{2}\right)=\frac{2}{17}\left\|r_{b}\right\|_{0, \omega_{\gamma_{c}}}^{2}
$$

which proves (4.5b).

Finally, using $p \in H^{1}(\Omega), \llbracket p \rrbracket_{e}=0$ a.e. on $e \in \mathcal{E}_{\mathcal{P}}$, the trace estimate (9.1) (see the appendix for a proof), and the fact that $q_{\mathcal{P}}$ is a piecewise constant function, we bound each jump as follows:

$$
\begin{aligned}
\frac{|K|}{\left|e_{j}\right|}\left\|\llbracket p-q_{\mathcal{P}} \rrbracket\right\|_{0, e_{j}}^{2} & \leq 2 \sum_{K: e_{j} \subset K}\left\|p-q_{\mathcal{P}}\right\|_{0, K}\left(\left\|p-q_{\mathcal{P}}\right\|_{0, K}+2\left|e_{i}\right|\left\|\boldsymbol{t}_{i} \cdot \nabla p\right\|_{0, K}\right) \\
& \leq 4 \sum_{K: e_{j} \subset K}\left(\left\|p-q_{\mathcal{P}}\right\|_{0, K}^{2}+\left|e_{i}\right|^{2}\left\|\boldsymbol{t}_{i} \cdot \nabla p\right\|_{0, K}^{2}\right)
\end{aligned}
$$

where $e_{i} \subset K$ is an incident edge to $e_{j}$ (i.e. $i=1, j=2$ or $i=2, j=1$ ). Then, we sum over the edges across which $s_{p}$ contains jumps and note for each $K \in \mathcal{P}$, that $s_{p}$ contains jumps across at least two and at most three different edges which proves (4.3).

We now present the main stability result.

Theorem 4.2. Let $s_{v}$ satisfy (3.5), let $\|\cdot\| \mid \|$ be defined by (4.1), and $s_{p}$ by (3.9) with $\alpha_{p} \geq \alpha$. Then,

$$
\sup _{(\boldsymbol{v}, q) \in \boldsymbol{V}_{1, \mathcal{P}} \times Q_{\mathcal{P}}} \frac{\mathfrak{B}_{s}((\boldsymbol{w}, r),(\boldsymbol{v}, q))}{\|(\boldsymbol{v}, q)\|} \geq \mu_{s}\|(\boldsymbol{w}, r)\| \quad \text { for all } \quad(\boldsymbol{w}, r) \in \boldsymbol{V}_{1, \mathcal{P}} \times Q_{\mathcal{P}},
$$

where $\mu_{s}=\beta_{G}^{2} /\left[2\left(1+\beta_{G}\right)\left(35+34 \beta_{G}\right)\right]$ where $\beta_{G}$ is the constant from (2.15). Hence, Problem (3.1) is well-posed.

Proof. Let $(\boldsymbol{w}, r) \in \boldsymbol{V}_{1, \mathcal{P}} \times Q_{\mathcal{P}}$ be given. First, from the definition of $\mathfrak{B}_{s}$ it follows that

$$
\mathfrak{B}_{s}((\boldsymbol{w}, r),(\boldsymbol{w},-r))=\|\boldsymbol{w}\|_{a+s}^{2}+s_{p}(r, r) .
$$

Additionally, given $\boldsymbol{w}_{\delta} \in \boldsymbol{V}_{1, \mathcal{P}}$, using (2.8), (3.5) and $\alpha:=1 /\left(c_{a}^{2}+c_{s}^{2}\right)$ we get

$$
\begin{aligned}
\mathfrak{B}_{s}\left((\boldsymbol{w}, r),\left(-\boldsymbol{w}_{\delta}, 0\right)\right) & =\left(a+s_{v}\right)\left(\boldsymbol{w},-\boldsymbol{w}_{\delta}\right)+\left(\operatorname{div} \boldsymbol{w}_{\delta}, r\right)_{\Omega} \\
& \geq-\sqrt{c_{a}^{2}+c_{s}^{2}}\|\boldsymbol{w}\|_{a+s}\left|\boldsymbol{w}_{\delta}\right|_{1, \Omega}+\left(\operatorname{div} \boldsymbol{w}_{\delta}, r\right)_{\Omega} \\
& \geq-\frac{1}{2}\|\boldsymbol{w}\|_{a+s}^{2}-\frac{1}{2 \alpha}\left|\boldsymbol{w}_{\delta}\right|_{1, \Omega}^{2}+\left(\operatorname{div} \boldsymbol{w}_{\delta}, r\right)_{\Omega} .
\end{aligned}
$$

Next, we choose $\boldsymbol{w}_{\delta}$. By (2.16) there exists $\boldsymbol{z} \in \boldsymbol{V}_{1, \mathcal{P}}$ such that $|\boldsymbol{z}|_{1, \Omega}=1$ and $(\operatorname{div} \boldsymbol{z}, r)_{\Omega} \geq \beta_{G}\|r\|_{0, \Omega}-\left(1+\beta_{G}\right)\left\|r-\Pi_{G} r\right\|_{0, \Omega}$. 
Defining $\boldsymbol{w}_{\delta}:=\delta \alpha\|r\|_{0, \Omega} \boldsymbol{z}$ with $\delta>0$ to be chosen, this last estimate, (4.2) and $\alpha \leq \alpha_{p}$ give

$$
\begin{aligned}
\left(\operatorname{div} \boldsymbol{w}_{\delta}, r\right)_{\Omega} & \geq \beta_{G} \delta \alpha\|r\|_{0, \Omega}^{2}-\left(1+\beta_{G}\right) \delta \alpha\|r\|_{0, \Omega} \alpha_{p}^{-1 / 2} C_{1}^{-1 / 2} s_{p}(r, r)^{1 / 2} \\
& \geq \beta_{G} \delta \alpha\|r\|_{0, \Omega}^{2}-\frac{\alpha}{2 C_{1}} \delta^{2}\left(1+\beta_{G}\right)^{2}\|r\|_{0, \Omega}^{2}-\frac{1}{2} s_{p}(r, r)
\end{aligned}
$$

and $\left|\boldsymbol{w}_{\delta}\right|_{1, \Omega}=\delta \alpha\|r\|_{0, \Omega}$ where $C_{1}=1 / 34$. We then define $(\boldsymbol{v}, q):=\left(\boldsymbol{w}-\boldsymbol{w}_{\delta},-r\right)$, and (4.14), (4.15) and (4.16) yield

$$
\begin{aligned}
\mathfrak{B}_{s}((\boldsymbol{w}, r),(\boldsymbol{v}, q)) & \geq \frac{1}{2}\left[\|\boldsymbol{w}\|_{a+s}^{2}+s_{p}(r, r)\right]+\left[\beta_{G}-\frac{\delta\left(1+\beta_{G}\right)^{2}}{2 C_{1}}\right] \delta \alpha\|r\|_{0, \Omega}^{2}-\frac{1}{2 \alpha}\left|\boldsymbol{w}_{\delta}\right|_{1, \Omega}^{2} \\
& =\frac{1}{2}\left[\|\boldsymbol{w}\|_{a+s}^{2}+s_{p}(r, r)\right]+\beta_{G}\left[1-\frac{\delta\left(1+\beta_{G}\right)^{2}}{2 C_{1} \beta_{G}}-\frac{\delta}{2 \beta_{G}}\right] \delta \alpha\|r\|_{0, \Omega}^{2} \\
& \geq \frac{\delta \beta_{G}}{2}\left(\|\boldsymbol{w}\|_{a+s}^{2}+s_{p}(r, r)+\alpha\|r\|_{0, \Omega}^{2}\right),
\end{aligned}
$$

where the choice $\delta:=\beta_{G} C_{1} /\left(C_{1}+\left(1+\beta_{G}\right)^{2}\right)=\beta_{G} /\left(1+34\left(1+\beta_{G}\right)^{2}\right)$ and $\delta \beta_{G} \leq 1$ imply the last estimate. On the other hand, using (2.8) and (3.5) shows $\|\boldsymbol{z}\|_{a+s} \leq \alpha^{-1 / 2}|\boldsymbol{z}|_{1, \Omega}$ for all $\boldsymbol{z} \in \boldsymbol{V}_{1, \mathcal{P}}$. Therefore, the definition of $\boldsymbol{w}_{\delta}$ and $\|\cdot\| \cdot \|$ give

$$
\|(\boldsymbol{v}, q)\| \leq\|(\boldsymbol{w}, r)\|+\left\|\boldsymbol{w}_{\delta}\right\|_{a+s} \leq\|(\boldsymbol{w}, r)\|+\delta \alpha^{1 / 2}\|r\|_{0, \Omega} \leq(1+\delta)\|(\boldsymbol{w}, r)\|,
$$

which proves the stated stability condition and the result with $\mu_{s}=\delta \beta_{G} /(2+2 \delta)$.

Remark 4.3. It is important to remark that the stability constant $\mu_{s}$ only depends on $\beta_{G}$, which is bounded below by a constant independent of the mesh aspect ratio. Therefore, $\mu_{s}$ is independent of the physical coefficients of the problem, and the aspect ratio of the triangulation. Furthermore, the stability estimate (4.13) is valid independently of the relation of $c_{a}$ and $c_{s}$. In [MT15] velocity stabilisation terms that satisfy (3.5) with $c_{s} \leq C c_{a}$ are used. We have chosen to avoid that assumption, since, as it has been shown in [JJ+14], a large stabilisation parameter in the grad-div term may be beneficial in some cases.

\section{A-priori estimates}

This section is devoted to the a-priori analysis of $(3.1)$. We use $\Pi_{Q_{\mathcal{P}}}: L^{2}(\Omega) \rightarrow Q_{\mathcal{P}}$ to denote the $L^{2}$-projection into $Q_{\mathcal{P}}$ satisfying

$$
\left(p-\Pi_{Q_{\mathcal{P}}} p, 1\right)_{K}=0 \text { for all } K \in \mathcal{P} .
$$

Theorem 5.1. Let us suppose the solution $(\boldsymbol{u}, p)$ of (2.2) satisfies $p \in H^{1}(\Omega)$. Let $s_{v}$ satisfy Assumption 3.1 and let $s_{p}$ be defined by (3.9) with $\alpha_{p} \geq \alpha$. Then, if $\boldsymbol{u}_{I} \in \boldsymbol{V}_{1, \mathcal{P}}$ is the interpolant defined in Lemma 2.4, then

$$
\begin{aligned}
& \|\|\left(\boldsymbol{u}-\boldsymbol{u}_{\mathcal{P}}, p-p_{\mathcal{P}}\right) \| \leq C\left(1+\mu_{s}^{-1}\right)\left\{s_{v}(\boldsymbol{u}, \boldsymbol{u})+s_{v}\left(\boldsymbol{u}-\boldsymbol{u}_{I}, \boldsymbol{u}-\boldsymbol{u}_{I}\right)+\sigma\left\|\boldsymbol{u}-\boldsymbol{u}_{I}\right\|_{0, \Omega}^{2}\right. \\
& +\sum_{K \in \mathcal{P}}\left(\left(\frac{1}{\alpha+\alpha_{p}}+\nu+\frac{b_{\infty, K}^{2} C_{\Omega}^{2}}{\nu+\sigma C_{\Omega}^{2}}\right)\left|\boldsymbol{u}-\boldsymbol{u}_{I}\right|_{1, K}^{2}\right. \\
& \left.\left.\quad+\left(\alpha+\alpha_{p}+\frac{1}{\nu+\gamma_{K}}\right)\left\|p-\Pi_{Q_{\mathcal{P}}} p\right\|_{0, K}^{2}+\alpha_{p} \sum_{i=1,2}\left|e_{i, K}\right|^{2}\left\|\partial_{t_{i}} p\right\|_{0, K}^{2}\right)\right\}^{1 / 2}, \quad \text { (5.2) }
\end{aligned}
$$


where $e_{i, K}, \partial_{t_{i}}(i=1,2)$ are defined as in Lemma 4.1, and the constant $C$ is independent of mesh and data.

Proof. As usual, we split the error as follows

$$
\left(\boldsymbol{u}-\boldsymbol{u}_{\mathcal{P}}, p-p_{\mathcal{P}}\right)=\left(\boldsymbol{u}-\boldsymbol{u}_{I}, p-\Pi_{Q_{\mathcal{P}}} p\right)-\left(\boldsymbol{u}_{\mathcal{P}}-\boldsymbol{u}_{I}, p_{\mathcal{P}}-\Pi_{Q_{\mathcal{P}}} p\right)=:\left(\boldsymbol{\eta}_{v}, \eta_{p}\right)-\left(\boldsymbol{\xi}_{v}, \xi_{p}\right) .
$$

Using (3.3) the interpolation error satisfies

$$
\left\|\left(\boldsymbol{\eta}_{v}, \eta_{p}\right)\right\|^{2}=\nu\left|\boldsymbol{\eta}_{v}\right|_{1, \Omega}^{2}+\sigma\left\|\boldsymbol{\eta}_{v}\right\|_{0, \Omega}^{2}+s_{v}\left(\boldsymbol{\eta}_{v}, \boldsymbol{\eta}_{v}\right)+\alpha\left\|\eta_{p}\right\|_{0, \Omega}^{2}+s_{p}\left(\eta_{p}, \eta_{p}\right) .
$$

The only term in the above expression that is not included in (5.2) is the last one. But, similar to (4.3), this term can be bounded by the last two in (5.2).

To bound the discrete error $\left(\boldsymbol{\xi}_{v}, \xi_{p}\right)$, using Theorem 4.2 there exists $\left(\boldsymbol{w}_{\mathcal{P}}, r_{\mathcal{P}}\right) \in \boldsymbol{V}_{1, \mathcal{P}} \times Q_{\mathcal{P}}$ with $\left\|\left(\boldsymbol{w}_{\mathcal{P}}, r_{\mathcal{P}}\right)\right\|=1$ satisfying

$$
\begin{aligned}
\mu_{s}\left\|\left(\boldsymbol{\xi}_{v}, \xi_{p}\right)\right\| & \leq \mathfrak{B}_{s}\left(\left(\boldsymbol{\xi}_{v}, \xi_{p}\right),\left(\boldsymbol{w}_{\mathcal{P}}, r_{\mathcal{P}}\right)\right) \\
& =\mathfrak{B}\left(\left(\boldsymbol{\eta}_{v}, \eta_{p}\right),\left(\boldsymbol{w}_{\mathcal{P}}, r_{\mathcal{P}}\right)\right)-s_{v}\left(\boldsymbol{u}_{I}, \boldsymbol{w}_{\mathcal{P}}\right)+s_{p}\left(\Pi_{Q_{\mathcal{P}}} p, r_{\mathcal{P}}\right),
\end{aligned}
$$

where we used (2.2) and (3.1). We estimate the right-hand side term by term. Using (3.7), a Cauchy-Schwarz estimate for $s_{p}(\cdot, \cdot)$, and $\left\|\left(\boldsymbol{w}_{\mathcal{P}}, r_{\mathcal{P}}\right)\right\|=1$ shows

$$
\begin{aligned}
& -s_{v}\left(\boldsymbol{u}_{I}, \boldsymbol{w}_{\mathcal{P}}\right)=s_{v}\left(\boldsymbol{\eta}_{v}, \boldsymbol{w}_{\mathcal{P}}\right)-s_{v}\left(\boldsymbol{u}, \boldsymbol{w}_{\mathcal{P}}\right) \leq s_{v}\left(\boldsymbol{\eta}_{v}, \boldsymbol{\eta}_{v}\right)^{1 / 2}+s_{v}(\boldsymbol{u}, \boldsymbol{u})^{1 / 2}, \\
& s_{p}\left(\Pi_{Q_{\mathcal{P}}} p, r_{\mathcal{P}}\right) \leq s_{p}\left(\Pi_{Q_{\mathcal{P}}} p, \Pi_{Q_{\mathcal{P}}} p\right)^{1 / 2},
\end{aligned}
$$

and applying $p \in H^{1}(\Omega)$ and (4.3) the right-hand sides of the last two inequalities are bounded by the first two and last two terms of (5.2). Next, using the Cauchy-Schwarz inequality and (2.7) we get

$$
\begin{aligned}
\nu\left(\nabla \boldsymbol{\eta}_{v}, \nabla \boldsymbol{w}_{\mathcal{P}}\right)_{\Omega}+\sigma\left(\boldsymbol{\eta}_{v}, \boldsymbol{w}_{\mathcal{P}}\right)_{\Omega} & \leq\left(\nu\left|\boldsymbol{\eta}_{v}\right|_{1, \Omega}^{2}+\sigma\left\|\boldsymbol{\eta}_{v}\right\|_{0, \Omega}^{2}\right)^{1 / 2}\left\|\boldsymbol{w}_{\mathcal{P}}\right\|_{a}, \\
\left((\boldsymbol{b} \cdot \nabla) \boldsymbol{\eta}_{v}, \boldsymbol{w}_{\mathcal{P}}\right)_{\Omega} & \leq\left(\sum_{K \in \mathcal{P}} b_{\infty, K}^{2}\left|\boldsymbol{\eta}_{v}\right|_{1, K}^{2}\right)^{1 / 2} \frac{C_{\Omega}}{\left(\nu+\sigma C_{\Omega}^{2}\right)^{1 / 2}}\left\|\boldsymbol{w}_{\mathcal{P}}\right\|_{a} .
\end{aligned}
$$

Moreover, for every $K \in \mathcal{P}$ we have

$$
\left(\operatorname{div} \boldsymbol{w}_{\mathcal{P}}, \eta_{p}\right)_{K} \leq \sqrt{2}\left|\boldsymbol{w}_{\mathcal{P}}\right|_{1, K}\left\|\eta_{p}\right\|_{0, K} .
$$

Alternatively, since $\left(\eta_{p},\left\langle\operatorname{div} \boldsymbol{w}_{\mathcal{P}}\right\rangle_{K}\right)_{K}=0$, we get

$$
\left(\operatorname{div} \boldsymbol{w}_{\mathcal{P}}, \eta_{p}\right)_{K}=\left(\kappa_{K}\left(\operatorname{div} \boldsymbol{w}_{\mathcal{P}}\right), \eta_{p}\right)_{K} \leq\left\|\kappa_{K}\left(\operatorname{div} \boldsymbol{w}_{\mathcal{P}}\right)\right\|_{0, K}\left\|\eta_{p}\right\|_{0, K}
$$

Then, using the inequality $a b \leq \sqrt{t}|a b|+\sqrt{1-t}|a b|$ with $t=\nu /\left(\nu+\gamma_{K}\right)$ to combine (5.6) and (5.7) leads to

$$
\left(\operatorname{div} \boldsymbol{w}_{\mathcal{P}}, \eta_{p}\right)_{K} \leq\left(\sqrt{2 \nu}\left|\boldsymbol{w}_{\mathcal{P}}\right|_{1, K}+\sqrt{\gamma_{K}}\left\|\kappa_{K}\left(\operatorname{div} \boldsymbol{w}_{\mathcal{P}}\right)\right\|_{0, K}\right)\left(\nu+\gamma_{K}\right)^{-1 / 2}\left\|\eta_{p}\right\|_{0, K} .
$$

Summing over all $K \in \mathcal{P}$ and employing (3.3), (3.4) and Assumption (3.6) we arrive at

$$
\left(\operatorname{div} \boldsymbol{w}_{\mathcal{P}}, \eta_{p}\right)_{\Omega} \leq C\left(\sum_{K \in \mathcal{P}} \frac{1}{\nu+\gamma_{K}}\left\|\eta_{p}\right\|_{0, K}^{2}\right)^{1 / 2}\left\|\boldsymbol{w}_{\mathcal{P}}\right\|_{a+s} .
$$


Finally, since $\Pi_{G} r_{\mathcal{P}} \in G$, we can apply (2.19) and (4.2) to conclude

$$
\begin{aligned}
\left(\operatorname{div} \boldsymbol{\eta}_{v}, r_{\mathcal{P}}\right)_{\omega} & =\left(\operatorname{div} \boldsymbol{\eta}_{v}, r_{\mathcal{P}}-\Pi_{G} r_{\mathcal{P}}\right)_{\omega} \\
& \leq \sqrt{2}\left|\boldsymbol{\eta}_{v}\right|_{1, \omega}\left\|r_{\mathcal{P}}-\Pi_{G} r_{\mathcal{P}}\right\|_{0, \omega} \leq\left. C \alpha_{p}^{-1 / 2}\left|\boldsymbol{\eta}_{v}\right|_{1, \omega} s_{p}\left(r_{\mathcal{P}}, r_{\mathcal{P}}\right)\right|_{\omega} ^{1 / 2}
\end{aligned}
$$

where $\omega=M \in \mathcal{M}$, or $\omega=M \cup M^{\prime}$ if $\gamma_{\boldsymbol{c}} \subset M \cap M^{\prime}$ for one $\gamma_{\boldsymbol{c}} \in \mathcal{E}_{\mathcal{E}}$. On the other hand, for any subset $\omega \subset \Omega$ we have

$$
\left(\operatorname{div} \boldsymbol{\eta}_{v}, r_{\mathcal{P}}\right)_{\omega} \leq\left\|\operatorname{div} \boldsymbol{\eta}_{v}\right\|_{0, \omega}\left\|r_{\mathcal{P}}\right\|_{0, \omega} \leq \alpha^{-1 / 2}\left|\boldsymbol{\eta}_{v}\right|_{1, \omega} \alpha^{1 / 2}\left\|r_{\mathcal{P}}\right\|_{0, \omega}
$$

Following the same steps as for (5.8), with $t=\alpha /\left(\alpha+\alpha_{p}\right)$ we combine (5.9) and (5.10) to arrive at

$$
\left(\operatorname{div} \boldsymbol{\eta}_{v}, r_{\mathcal{P}}\right)_{\Omega} \leq C\left(\sum_{K \in \mathcal{P}} \frac{1}{\alpha+\alpha_{p}}\left\|\boldsymbol{\eta}_{v}\right\|_{0, K}^{2}\right)^{1 / 2}\left(\alpha\left\|r_{\mathcal{P}}\right\|_{0, \Omega}^{2}+s_{p}\left(r_{\mathcal{P}}, r_{\mathcal{P}}\right)\right)^{1 / 2}
$$

The result follows on collecting the estimates (5.3)-(5.5), (5.8), and (5.11).

We close this section with a few remarks on Theorem 5.1:

1) A different proof of (5.2) also implies a best approximation result. More precisely, if the property (2.19) of $\boldsymbol{u}_{I}$ (and hence (5.9)) is not used, then the terms involving $\boldsymbol{u}-\boldsymbol{u}_{I}$ become

$$
\inf _{\boldsymbol{v}_{\mathcal{P}} \in \boldsymbol{V}_{1, \mathcal{P}}}\left|\boldsymbol{u}-\boldsymbol{v}_{\mathcal{P}}\right|_{s_{v}}^{2}+\sum_{K \in \mathcal{P}}\left(\frac{1}{\alpha}+\nu+\frac{b_{\infty, K}^{2} C_{\Omega}^{2}}{\nu+\sigma C_{\Omega}^{2}}\right)\left|\boldsymbol{u}-\boldsymbol{v}_{\mathcal{P}}\right|_{1, K}^{2}+\sigma\left\|\boldsymbol{u}-\boldsymbol{v}_{\mathcal{P}}\right\|_{0, K}^{2}
$$

This result extends, for instance, [MT15, Theorem 4.4] to the non-inf-sup stable pair $\boldsymbol{V}_{1, \mathcal{P}} \times Q_{\mathcal{P}}$ on anisotropic meshes. This bound, as well as the one in Theorem 5.1, does show a dependency on $\nu$ that could be harmful if $\sigma$ vanishes. It is important to remark though that this dependence is common to many different discretisations of the Oseen equations, even for isotropic meshes. In particular, the present error estimate is qualitatively similar to the ones presented in the review paper $[\mathrm{BB}+07]$ for different discretisations of the Oseen problem, and to some some of the estimates presented in [Cod08]. One way to avoid this could be to use the technique developed in [Bra08] for the $\mathbb{Q}_{1}^{2} \times \mathbb{Q}_{1}$ pair. In there, the method is defined by penalising the fluctuations of the whole gradient (instead of the convective gradient, as in this work). The downside of that approach is the fact that the corresponding norm does not control $\|p\|_{0, \Omega}$ (see also the appendix in [MPP03] for a similar issue) and the a-priori estimates require $p \in H^{2}(\Omega)$.

2) The choice $\alpha_{p} \geq \alpha$ is motivated by the fact that it leads to stability constants which are independent of the data of the problem. Moreover, the inclusion of the pressure stabilisation term in the energy norm allows an error estimate containing the factor $\left(\alpha+\alpha_{p}\right)^{-1}$. This is a better bound than $1 / \alpha$, which for $\sigma=0$ behaves like $\nu^{-1}$.

3) The error estimate contains $s_{v}\left(\boldsymbol{\eta}_{v}, \boldsymbol{\eta}_{v}\right)^{1 / 2}$ and $s_{v}(\boldsymbol{u}, \boldsymbol{u})^{1 / 2}$, which are, generally, significantly smaller than their crude bound $c_{s}\left|\boldsymbol{\eta}_{v}\right|_{1, \Omega}$ (especially for the pure grad-div term). This, as well, provides more flexibility for the choice of $\gamma_{K}$ (see [JJ+14] for a detailed discussion of this issue in the case of the Stokes problem). 
4) Alternatively, a mixed method could be proposed using $\boldsymbol{V}_{1, \mathcal{P}} \times G$ as an approximation space. In such a case, the proof of a priori estimate (5.2) changes, since $\Pi_{Q_{\mathcal{P}}}$ is replaced by $\Pi_{G}$. Hence, (5.7) requires Assumption (3.6) to be modified accordingly. More precisely, we observe that $G$ has a locally constant basis $\left\{\phi_{j}\right\}_{j=1}^{\operatorname{dim} G}$ and define $\omega_{j}:=\operatorname{supp} \phi_{j}$, where either $\omega_{j}=M$ or $\omega_{j}=M \cup M^{\prime}$ with $M, M^{\prime} \in \mathcal{M}$. Now, defining

$$
s_{v}^{G}(\boldsymbol{u}, \boldsymbol{v}):=\sum_{j=1}^{\operatorname{dim} G} \gamma_{\omega_{j}}\left(\kappa_{\omega_{j}}(\operatorname{div} \boldsymbol{u}), \kappa_{\omega_{j}}(\operatorname{div} \boldsymbol{v})\right)_{\omega_{j}},
$$

we can replace assumption $(3.6)$ by $s_{v}^{G}(\boldsymbol{v}, \boldsymbol{v}) \leq s_{v}(\boldsymbol{v}, \boldsymbol{v})$. The latter definitions directly imply (5.7) with $\kappa_{\omega_{j}}$ instead of $\kappa_{K}$. Then, (5.8) changes to

$$
\left(\operatorname{div} \boldsymbol{w}_{\mathcal{P}}, \eta_{p}\right)_{\Omega} \leq C\left(\sum_{j=1}^{\operatorname{dim} G} \frac{1}{\nu+\gamma_{\omega_{j}}}\left\|p-\Pi_{G} p\right\|_{0, \omega_{j}}^{2}\right)^{1 / 2}\left\|\boldsymbol{w}_{\mathcal{P}}\right\|_{a+s} .
$$

On the other hand, estimate (5.9) is not needed as $\left(\operatorname{div} \boldsymbol{\eta}_{v}, r_{G}\right)_{\omega}=0$ by definition.

\section{Examples of stabilisation terms for the velocity}

The previous sections, in particular Section 3, leave the choice of velocity stabilisation terms flexible. Below we define the stabilisation terms used in the numerical experiments.

Option one. Let $\boldsymbol{b}_{K}:=\langle\boldsymbol{b}\rangle_{K}$, and define

$$
\begin{aligned}
s_{v}(\boldsymbol{u}, \boldsymbol{v}):= & \sum_{M \in \mathcal{M}} \gamma_{M}\left(\kappa_{M}(\operatorname{div} \boldsymbol{u}), \kappa_{M}(\operatorname{div} \boldsymbol{v})\right)_{M} \\
& +\sum_{K \in \mathcal{P}} \frac{L}{\left|\boldsymbol{b}_{K}\right|}\left(\kappa_{K}\left(\left(\boldsymbol{b}_{K} \cdot \nabla\right) \boldsymbol{u}\right), \kappa_{K}\left(\left(\boldsymbol{b}_{K} \cdot \nabla\right) \boldsymbol{v}\right)\right)_{K} .
\end{aligned}
$$

Here, $\gamma_{M}$ is chosen as one of the following options

$$
\begin{aligned}
\gamma_{M} & :=\max \left\{1, \mathrm{Pe}_{\mathcal{M}}^{\min }\right\} \\
\gamma_{M} & :=1+\operatorname{ind}(M) \mathrm{Pe}_{M}^{\min } \quad \text { and } \quad \operatorname{ind}(M):=1-\frac{\rho_{M}|M|}{\max _{\omega \in \mathcal{M}}|\omega|},
\end{aligned}
$$

with local and global (minimal) Péclet numbers defined by $\mathrm{Pe}_{\mathcal{M}}^{\min }:=\min _{M \in \mathcal{M}} \mathrm{Pe}_{M}^{\min }$ and $\mathrm{Pe}_{M}^{\min }:=\nu^{-1} b_{\infty, M} \min \{|M| /|e|: e$ is an edge of $M\}$. In addition, for dimensional consistency, we have included the length scale $L$. We choose a characteristic length scale which is related to the domain $\Omega$, i.e., it is global. This choice is motivated by two main reasons: the stability and convergence analyses do not require a local length scale, and our numerical experiments show that a better behavior arises when the value of $L$ does not tend to zero with any mesh properties.

The choice $(6.2 \mathrm{~b})$ is motivated by the fact that the minimal global Péclet number does not contain information about local phenomena. Then the introduction of the $\operatorname{ind}(\cdot)$ function ensures that $\gamma_{M}$ varies significantly with local geometric properties of $M$. In fact $\gamma_{M} \approx 1$ in large shape-regular elements and $\gamma_{M} \approx 1+\mathrm{Pe}_{M}^{\min }$ in highly stretched elements and small corner elements, which is the desirable behaviour. 
Remark 6.1. The stabilisation term (6.1) satisfies Assumption 3.1; moreover, estimate (3.5) follows with with $c_{s}=\left(b_{\infty, \Omega}+\max _{M \in \mathcal{M}} 2 \gamma_{M}\right)^{1 / 2}$. In addition, the definition (6.1) of $s_{v}$ guarantees that $s_{v}(\boldsymbol{u}, \boldsymbol{u})$ and $s_{v}\left(\boldsymbol{\eta}_{u}, \boldsymbol{\eta}_{u}\right)$, appearing in the a-priori estimate (5.2), can be bounded in an optimal way.

Option two. We also consider the following stabilisation

$$
s_{v}(\boldsymbol{u}, \boldsymbol{v}):=\sum_{M \in \mathcal{M}}\left(\kappa_{M}\left(\partial_{x} \boldsymbol{u}\right), \delta_{x} \kappa_{M}\left(\partial_{x} \boldsymbol{v}\right)\right)_{M}+\left(\kappa_{M}\left(\partial_{y} \boldsymbol{u}\right), \delta_{y} \kappa_{M}\left(\partial_{y} \boldsymbol{v}\right)\right)_{M},
$$

where $\left(\delta_{x}, \delta_{y}\right)$ are given by

$$
\begin{aligned}
\delta_{K, x} & :=\nu^{-1} b_{\infty, K}^{2} h_{K, x}^{2} \min \left\{1,\left(\mathrm{Pe}_{K}^{\min }\right)^{-1}\right\}, \\
\delta_{K, y} & :=\nu^{-1} b_{\infty, K}^{2} h_{K, y}^{2} \min \left\{1,\left(\mathrm{Pe}_{K}^{\min }\right)^{-1}\right\}, \\
\mathrm{Pe}_{K}^{\min } & :=\nu^{-1} \min \left\{h_{K, x}, h_{K, y}\right\} b_{\infty, K} .
\end{aligned}
$$

This term has been introduced and analysed in [Bra08] in the context of the $\mathbb{Q}_{1}^{2} \times \mathbb{Q}_{1}$ pair. It satisfies Assumption 3.1 with $c_{s}=\max \left\{\delta_{x}, \delta_{y}\right\}^{1 / 2}$ and $\gamma_{K}=\gamma_{M}:=\frac{1}{2} \min \left\{\delta_{x}, \delta_{y}\right\}$.

\section{Numerical verification}

In this section we report numerical results confirming our theoretical findings. In all numerical experiments presented below the domain is chosen to be $\Omega=(0,1)^{2}$. This is why we have chosen as characteristic length scale the value $L=1$.

\subsection{Numerical stability}

This experiment's aim is to show the impact of the addition of the stabilising term (3.9c) in the formulation. For this, we have used Lemma 9.2 below to compute the infsup constant of the bilinear form $\mathfrak{B}_{s}((\cdot, \cdot),(\cdot, \cdot))$ using $\alpha_{p}=1$. The physical coefficients of the problem are $\boldsymbol{b}=(-1,-1)^{\top}, \nu=1, \sigma=1$, and we impose homogeneous Dirichlet boundary conditions. The stabilising term $s_{p}(\cdot, \cdot)$ has been implemented either in its complete form, i.e., including (3.9c), or dropping that term. Since we aim to asses the impact of $(3.9 \mathrm{c})$ on the stability of the method, we have set $s_{v}:=0$ for this experiment. The results for the corner patches $\mathcal{P}_{0}, \mathcal{P}_{1}, \mathcal{P}_{2}$ shown in Figure 2 are depicted in Figure 6 where we observe that the presence of $(3.9 \mathrm{c})$ helps obtaining a stability constant $\mu_{s}$ which is bounded below independently of $\lambda$, while the inf-sup constant of the method without the term (3.9c) degenerates as the aspect ratio tends to zero.
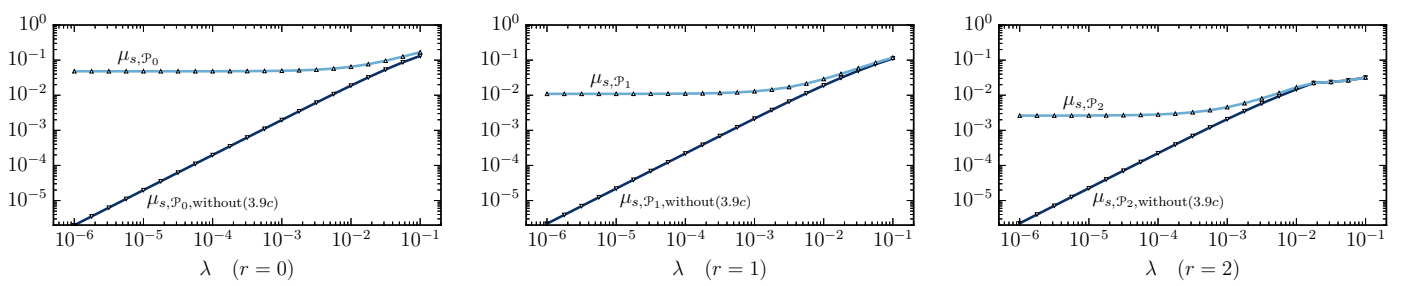

Fig. 6 Constants $\mu_{s}$ and $\mu_{s \text {,without (3.9c) }}$, for $r=0,1,2$. 


\subsection{Quality of approximations}

We present the results of two different experiments approximating a solution of (2.1) with non-homogeneous boundary conditions.

Example 1. We define $\boldsymbol{b}=(-1,-1)^{\top}, \nu=10^{-6}, \sigma=0$ and choose the right-handside $\boldsymbol{f}$ and boundary conditions such that the exact solution is given by

$$
\boldsymbol{u}:=\left(\begin{array}{l}
\frac{1-\exp (-y / \nu)}{1-\exp (-1 / \nu)}-y \\
\frac{1-\exp (-x / \nu)}{1-\exp (-1 / \nu)}-x
\end{array}\right) \quad \text { and } \quad p:=\sin (x-1 / 2) \sin (y-1 / 2) .
$$

Example 2. We define $\boldsymbol{b}=(-1,-1)^{\top}, \nu=10^{-6}, \sigma=1$ and choose the right-handside $\boldsymbol{f}$ and boundary conditions such that the exact solution is given by

$$
\boldsymbol{u}:=\left(\begin{array}{l}
1-\exp \left(-y \frac{1+\sqrt{1+4 \nu}}{2 \nu}\right) \\
1-\exp \left(-x \frac{1+\sqrt{1+4 \nu}}{2 \nu}\right)
\end{array}\right) \quad \text { and } \quad p:=\sin (x-1 / 2) \sin (y-1 / 2) .
$$

In both cases the right-hand-side $f$ is independent of $\nu$, which makes the results independent of the quadrature rules employed.

For the experiments we define parametrised partitions containing a corner patch. Let $\mathcal{P}_{N, \lambda}$ ( $N$ divisible by 4 , and $\left.\lambda \in(0,1 / 2]\right)$ be the tensor-product of the one-dimensional interval subdivision that splits each of the intervals $[0, \lambda]$ and $[\lambda, 1]$ into $N / 2$ intervals of equal length, cf. Figure 1 (right) where $\mathcal{P}_{4, \lambda}$ is shown. The mesh $\mathcal{P}_{N, \lambda}$ is a Shishkin mesh, but we choose $\lambda$ to be larger than the Shishkin parameter $2 \nu \ln N \leq 10^{-5}$.

Our aim is to explore how robust the methods with the previously defined stabilisation terms and parameters are with respect to the choice of $\lambda$. This is why we chose a wide range for $\lambda$ from $\lambda=1 / 2$ (a shape-regular mesh) to $\lambda=10^{-4}$ (a highly anisotropic corner patch with minimal aspect ratio $\left.\varrho \approx 10^{-4}\right)$.

The first study concerns the error behaviour of the discretisation error when compared to a reference value. To this end, we compute the relative errors given by

$$
E_{n a t}^{r e l}:=\frac{\left\|\left(\boldsymbol{u}-\boldsymbol{u}_{\mathcal{P}}, p-p_{\mathcal{P}}\right)\right\|}{I E_{\text {nat }}}, \quad \text { with } \quad I E_{\text {nat }}:=\left\|\left(\boldsymbol{u}-I_{\mathcal{P}} \boldsymbol{u}, p-\Pi p\right)\right\|,
$$

where $\|\cdot\| \|$ is defined in (4.1). Here $I_{\mathcal{P}} \boldsymbol{u} \in \boldsymbol{V}_{1, \mathcal{P}}$ stands for the nodal interpolant of $\boldsymbol{u}$, and $\Pi \in\left\{\Pi_{Q_{\mathcal{P}}}, \Pi_{G}\right\}$ are the projections defined earlier, chosen depending on whether we use the stabilised method based on $\boldsymbol{V}_{1, \mathcal{P}} \times Q_{\mathcal{P}}$, or the inf-sup stable pair $\boldsymbol{V}_{1, \mathcal{P}} \times G$, respectively.

Furthermore, since we compare different stabilisation terms for velocity, we define $E_{n a t, 2 a}^{r e l}$ when $s_{v}=(6.1) \&(6.2 \mathrm{a}), E_{n a t, 2 b}^{r e l}$ when $s_{v}=(6.1) \&(6.2 \mathrm{~b})$ and $E_{n a t, 3}^{r e l}$ when $s_{v}=(6.3)$. For the test cases performed, we notice that the nodal interpolation of the exact velocity is divergence-free. Then, the error $I E_{n a t}$ is independent of the stabilisation parameter in the case we use the stabilisation term given by (6.1).

Qualitatively similar results have been observed for both Ex. 1 and Ex. 2. Then, we only show results for Ex. 2 . In Table 2 we report the results using $\lambda=10^{-4}$. The results reported in Table 2 show that the discretisation error, for all the cases tested, follows the same pattern as the interpolation error. In addition, we observe that both errors tend to zero as $N$ grows, which is linked to the fact that $\lambda$ is small enough so the boundary layer becomes resolved as $N$ grows. It is interesting to remark that the natural norm 
TABLE 2. Approximation errors for Solution (7.2), on meshes with $\lambda=10^{-4}$. Errors using the inf-sup stable pair $\boldsymbol{V}_{1, \mathcal{P}} \times G$ (top table). Errors using $\boldsymbol{V}_{1, \mathcal{P}} \times Q_{\mathcal{P}}, \alpha_{p}=1$, with $s_{v}$ given by (6.3) (bottom left) and (6.1) (bottom right).

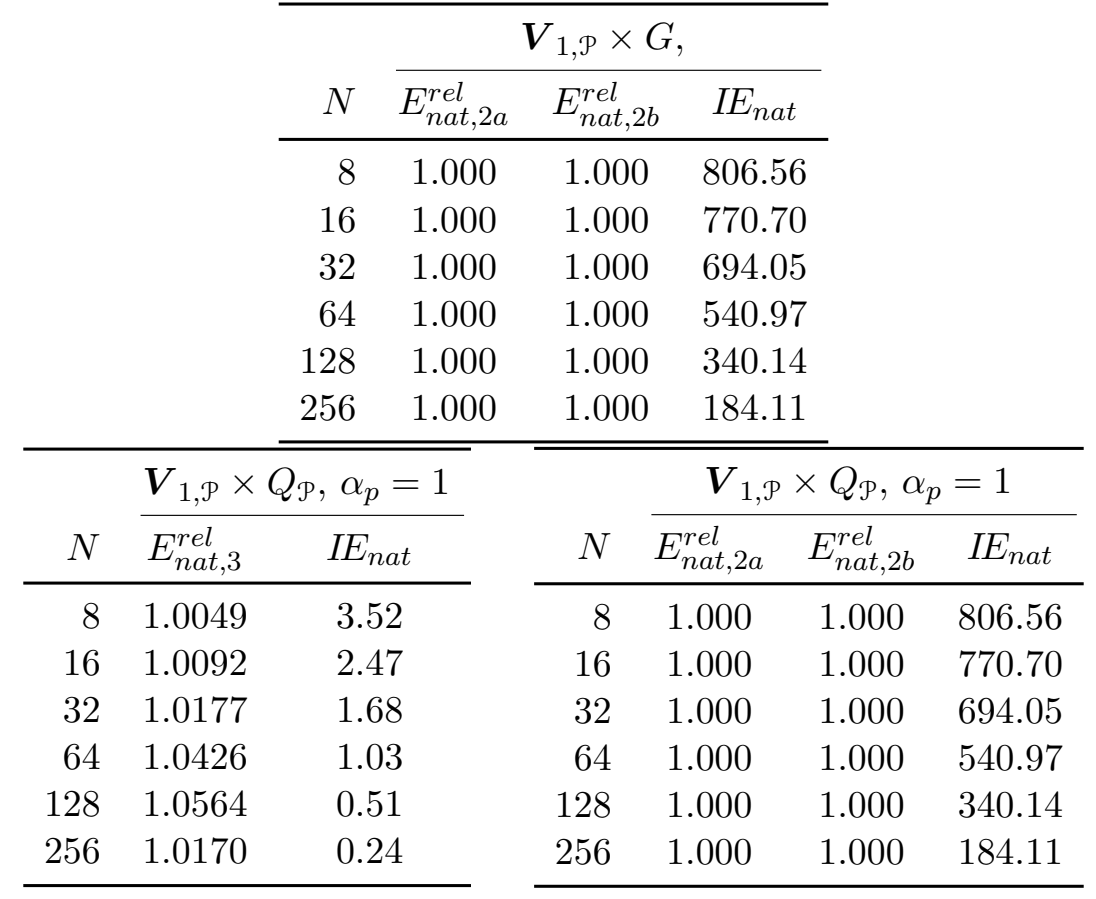

is much stronger for the case in which $s_{v}$ is given by (6.1) (for both definitions of the stabilisation parameter) than for the case of $s_{v}$ given by (6.3).

We next try to assess the importance of the last point made in the last paragraph. For this, we plot the velocity profiles obtained by the different methods in in Figures 78. We note that the stabilisation term $s_{v}$ given by (6.1) produces a profile which is smoother, especially when $(6.2 \mathrm{a})$ is used, producing a discrete velocity which is almost free of oscillations, whereas the profile obtained from the method using $s_{v}$ given by (6.3) presents large oscillations. This is in accordance with our earlier claim that the norm induced by (6.1) is stronger than the one induced by (6.3). Furthermore, from Figures 78 we see that the different definitions of the stabilisation parameters give different results on shape regular meshes (see the results for $\lambda=1 / 2$ ). However, on anisotropic meshes the behaviour is similar and oscillations and overshoots are significantly reduced.

We finally show that both terms in (6.1) are necessary for the good behavior of the method. For this, we remove the second term from (6.1), i.e., the fluctuations of the convective gradient, and solve the discrete problem with this "reduced" stabilised method. The results are depicted in Figure 9 where we can observe that the "reduced" method (depicted on the left) presents oscillations in the discrete solution, which are corrected once the full stabilising term $s_{v}$ given by $(6.1)$ is used. This result indicate that both terms in the stabilising term are necessary, although the fluctuation term in the convective derivative seems to only be active in the region close to the boundary layers. Then, the main responsible for the stable velocity profiles seems to be the fluctuations of the divergence term. 


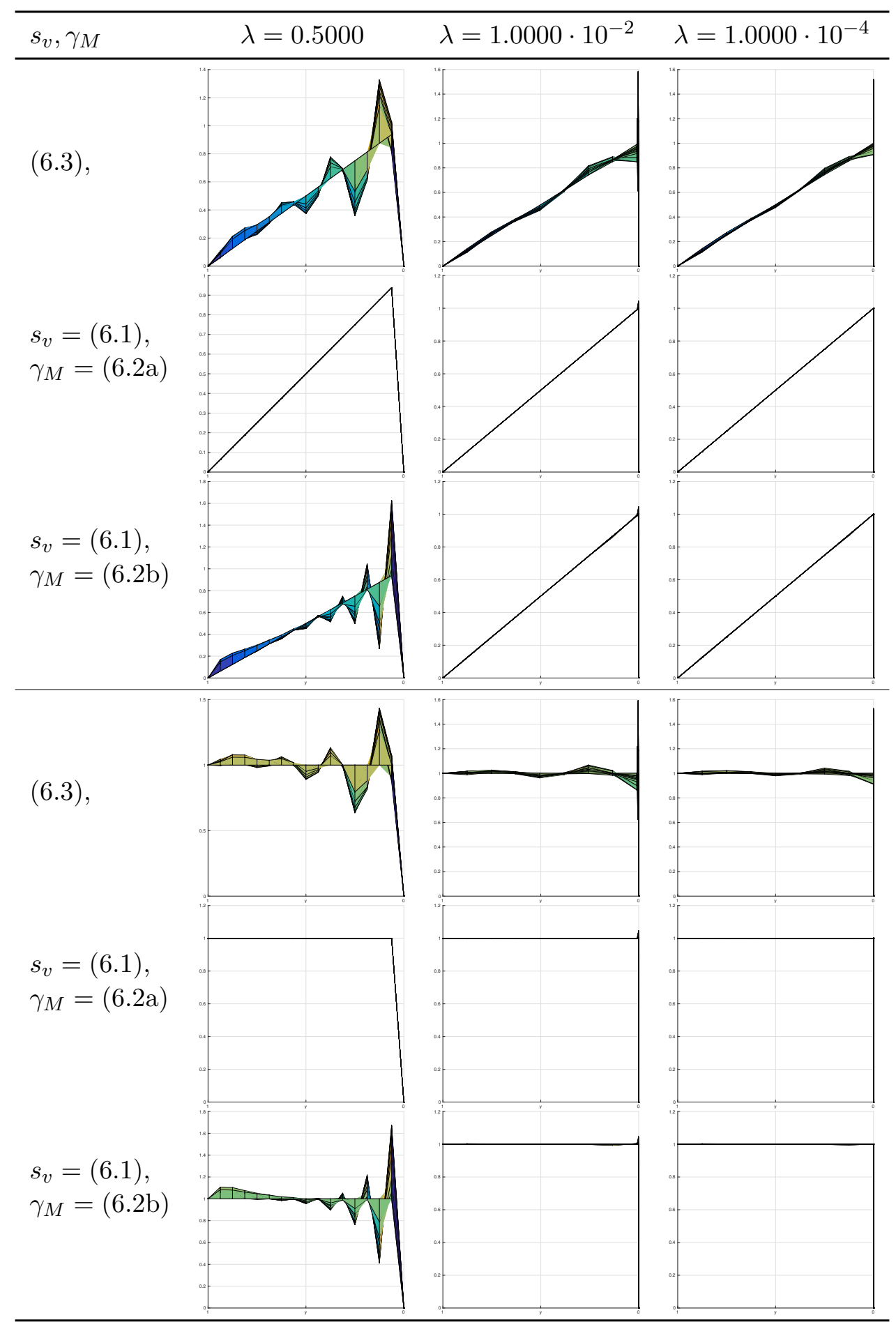

FIG. 7 Velocity profiles using $N=16$, and the mixed method $\boldsymbol{V}_{1, \mathcal{P}} \times G$. At the top we depict the results for Example 1, while at the bottom the results correspond to Example 2. 


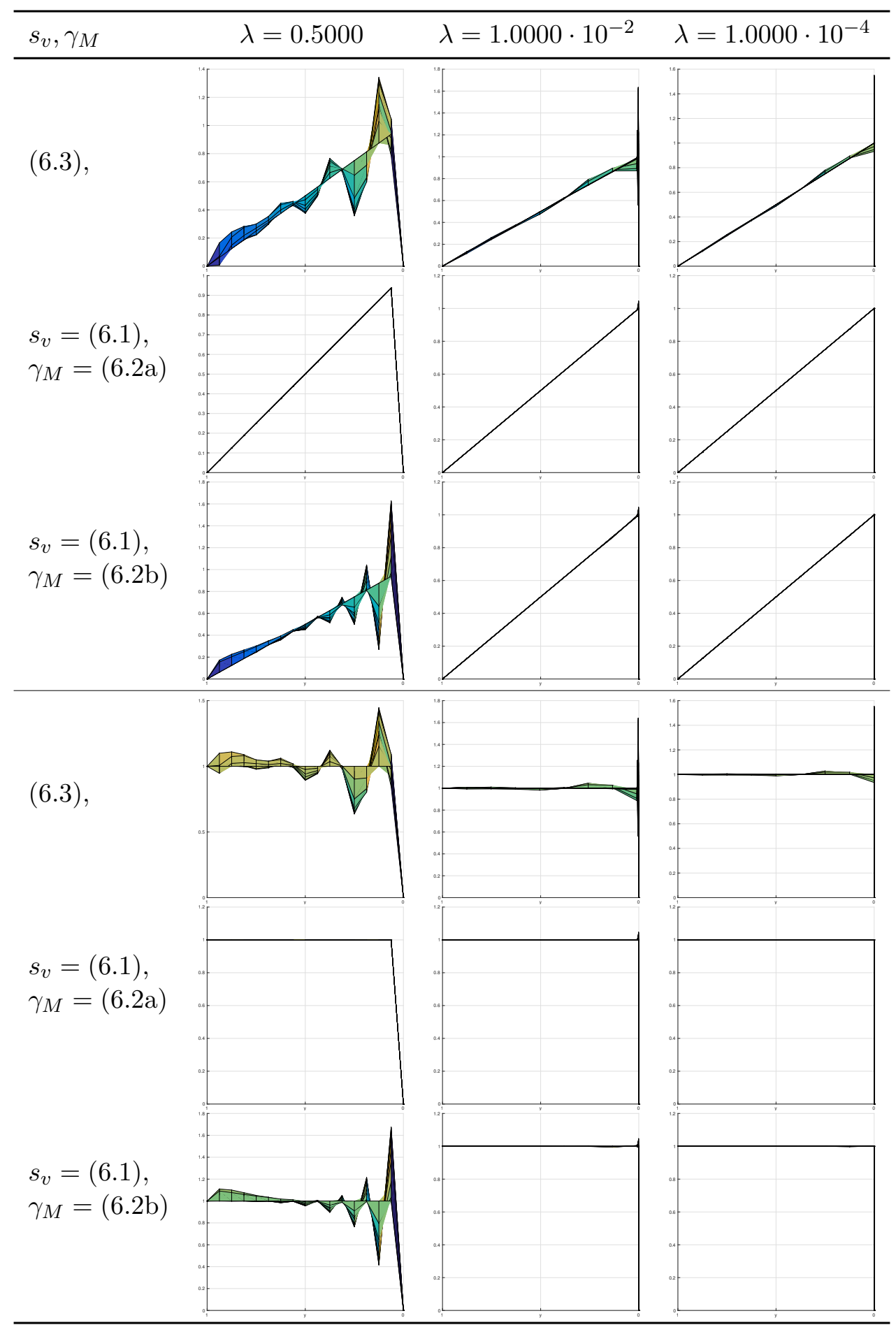

FIG. 8 Velocity profiles using $N=16$ and the stabilised method with $\boldsymbol{V}_{1, \mathcal{P}} \times Q_{\mathcal{P}}, s_{p}$, and $\alpha_{p}=1$. At the top we depict the results for Example 1, while at the bottom the results correspond to Example 2. 

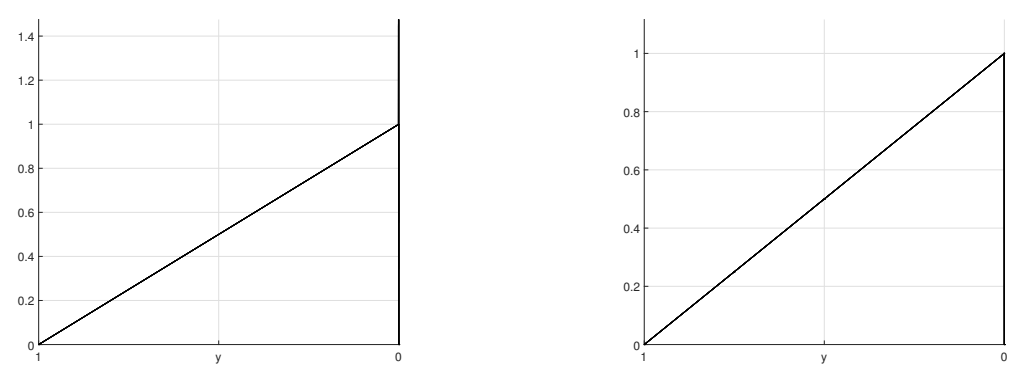

FIG. 9 Discrete velocities for Example 1 on a mesh with $N=8, \lambda=10^{-3}$. The method uses $\boldsymbol{V}_{1, \mathcal{P}} \times Q_{\mathcal{P}}, s_{p}$, and $\alpha_{p}=1$, and the "reduced" stabilised method (i.e., (6.1) where the fluctuation of the convective derivative has been removed, depicted on the left), and the full stabilising term (6.1), depicted on the right.

\section{Conclusion}

In this work we have generalised the results from [ABW15, LS13] to the lowest order pair $\mathbb{Q}_{1}^{2} \times \mathbb{P}_{0}$ in partitions that contain refined corner patches, and extended this generalisation to the Oseen equation. To analyse the resulting methods we have used, and adapted when necessary, the abstract approach given in [MT15]. This allowed us to present stability and convergence results that are valid both in the inf-sup stable and stabilised cases. A precise definition, by means of a weighted grad-div term enhanced by a penalisation of the convective derivative, of the stabilisation term for the velocity has been proposed, justified, and tested numerically. This new definition seems to outperform some previously known alternatives, at least numerically. This study leaves, nevertheless, some open questions such as the extension of this idea to graded meshes, more general quadrilaterals, and the three-dimensional case. For the latter, the detailed stability analysis of the underlining $\boldsymbol{V}_{1, \mathcal{P}} \times Q_{\mathcal{P}}$ space needs to be done. These will be the topics of future research.

\section{Acknowledgement}

AW wants to acknowledge the partial support given by the Asociación Mexicana de Cultura A.C.. The authors want to thank Jen Pestana for helpful discussions related to discrete inf-sup constants. The authors also want to thank the anonimous referees for their careful reading of the paper and their useful suggestions.

\section{Appendix}

In this appendix we detail a local trace inequality. Its proof does not seem to be available, and that is why we present it here.

Lemma 9.1. Let $K$ be a parallelogram, let e be one of its edges, and let $\boldsymbol{t}_{i}$ be a unit tangential vector of an edge $e_{i}$ that is incident to $e$. Then, for all $v \in H^{1}(K)$ the following holds

$$
\|v\|_{0, e}^{2} \leq \frac{|e|}{|K|}\|v\|_{0, K}\left(\|v\|_{0, K}+2\left|e_{i}\right|\left\|\boldsymbol{t}_{i} \cdot \nabla v\right\|_{0, K}\right) .
$$


Proof. Let $v \in H^{1}(K)$. Using the notation given by Figure 10, we define

$$
\phi_{e}(\boldsymbol{x}):=\left(\boldsymbol{n}_{e} \cdot\left(\boldsymbol{x}-\boldsymbol{p}_{\bar{e}}\right)\right) \boldsymbol{t}_{i},
$$

where $\boldsymbol{n}_{e}$ is the outer unit normal to edge $e$ and $\boldsymbol{p}_{\overline{\bar{e}}}$ is any point on the opposite edge $\overline{\bar{e}}$ (parallel to $e$ ). Since $\phi_{e} \in C^{\infty}(K)$, the following Green's formula holds

$$
\int_{\partial K}\left(\phi_{e} \cdot \boldsymbol{n}\right) v^{2} \mathrm{~d} s=\int_{K} v^{2} \operatorname{div} \phi_{e} \mathrm{~d} \boldsymbol{x}+2 \int_{K} v\left(\phi_{e} \cdot \nabla v\right) \mathrm{d} \boldsymbol{x} .
$$

We now evaluate the terms involving $\phi_{e}$. We start by observing that

$$
\operatorname{div} \phi_{e}=n_{e 1} t_{i 1}+n_{e 2} t_{i 2}=\boldsymbol{n}_{e} \cdot \boldsymbol{t}_{i}=\cos (\pi-(\pi / 2+\alpha))=\sin (\alpha) .
$$

Furthermore, the unit tangential and normal vector of edge $e$ form a basis of $\mathbb{R}^{2}$, that is, each $\boldsymbol{x} \in K$ is representable as $\boldsymbol{x}=\boldsymbol{p}_{\overline{\bar{e}}}+r_{1}(\boldsymbol{x})|e|^{-1}|K| \boldsymbol{n}_{e}+r_{2}(\boldsymbol{x}) \boldsymbol{t}_{e}$ with $r_{1} \in[0,1]$ and $r_{2} \in \mathbb{R}$. Therefore, the definition of $\phi_{e}$ simplifies to

$$
\phi_{e}=r_{1}(\boldsymbol{x})|e|^{-1}|K| \boldsymbol{t}_{i} \quad \text { for } \quad r_{1} \in[0,1]
$$

with $r_{1} \equiv 0$ on $\overline{\bar{e}}$ and $r_{1} \equiv 1$ on $e$ (since $K$ is a parallelogram). Therefore, $\phi_{e} \cdot \boldsymbol{n}=0$ on $\partial K \backslash e$ and

$$
\int_{\partial K}\left(\phi_{e} \cdot \boldsymbol{n}\right) v^{2} \mathrm{~d} s=\int_{e}\left(\phi_{e} \cdot \boldsymbol{n}_{e}\right) v^{2} \mathrm{~d} s=\frac{|K|}{|e|}\left(\boldsymbol{n}_{e} \cdot \boldsymbol{t}_{i}\right) \int_{e} v^{2} \mathrm{~d} s=\frac{|K|}{|e|} \sin (\alpha) \int_{e} v^{2} \mathrm{~d} s .
$$

Then, inserting (9.3), (9.4) and (9.5) into (9.2) we arrive at

$$
\sin (\alpha) \frac{|K|}{|e|} \int_{e} v^{2} \mathrm{~d} s=\sin (\alpha) \int_{K} v^{2} \mathrm{~d} \boldsymbol{x}+\frac{|K|}{|e|} \int_{K} r_{1}(\boldsymbol{x}) 2 v\left(\boldsymbol{t}_{i} \cdot \nabla v\right) \mathrm{d} \boldsymbol{x} .
$$

After recalling $\max _{\boldsymbol{x} \in K} r_{1}(\boldsymbol{x})=1$ and applying Cauchy-Schwarz's inequality the last identity gives

$$
\begin{aligned}
\|v\|_{0, e}^{2} & \leq\|v\|_{0, K}\left(\frac{|e|}{|K|}\|v\|_{0, K}+\frac{2}{\sin (\alpha)}\left\|\boldsymbol{t}_{i} \cdot \nabla v\right\|_{0, K}\right) \\
& =\frac{|e|}{|K|}\|v\|_{0, K}\left(\|v\|_{0, K}+2\left|e_{i}\right|\left\|\boldsymbol{t}_{i} \cdot \nabla v\right\|_{0, K}\right),
\end{aligned}
$$

which finishes the proof.

We now prove a result that allows us to compute the inf-sup constant of the Oseen problem. We have not been able to locate this exact result in the literature, and that is why we detail its proof here.

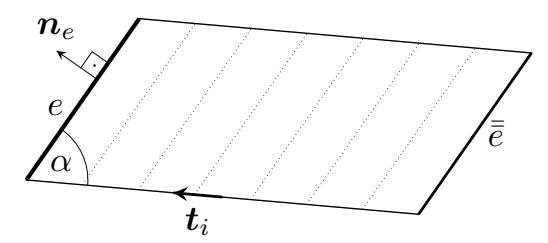

Fig. 10 Parallelogram $K$ with notation and level-sets of $\phi_{e}$. 
Lemma 9.2. Let $A, B \in \mathbb{R}^{n \times n}$ and let $B$ be symmetric positive definite. Then, the inf-sup constant $\sigma$ defined by

$$
\sigma=\min _{z \in \mathbb{R}^{n}} \max _{\xi \in \mathbb{R}^{n}} \frac{\xi^{T} A z}{\|\xi\|_{B}\|z\|_{B}}
$$

is given by the smallest singular value of $\widetilde{A}:=L^{-T} A L^{-1}$ where $L$ is defined by the Cholesky decomposition $B=L^{T} L$.

Proof. First, a direct application of Theorem 2 in [CF03] implies

$$
\sigma\|y\|_{2} \leq \max _{x \in \mathbb{R}^{n}} \frac{x^{T} \widetilde{A} y}{\|x\|_{2}} \quad \text { for all } y \in \mathbb{R}^{n},
$$

where $\sigma$ is the smallest singular value of $\widetilde{A}$. Then, changing variables $(x=L \xi, y=L z)$ we get

$$
\sigma\|L z\|_{2} \leq \max _{\xi \in \mathbb{R}^{n}} \frac{(L \xi)^{T} L^{-T} A L^{-1}(L z)}{\|L \xi\|_{2}}=\max _{\xi \in \mathbb{R}^{n}} \frac{\xi^{T} A z}{\|L \xi\|_{2}} \quad \text { for all } \quad z \in \mathbb{R}^{n} .
$$

Finally, realising $\|z\|_{B}^{2}=\langle B z, z\rangle=\left\langle L^{T} L z, z\right\rangle=\langle L z, L z\rangle=\|L z\|_{2}^{2}$ finishes the proof.

\section{References}

[ABV06] R. Araya, G. R. Barrenechea, and F. Valentin, Stabilized finite element methods based on multiscale enrichment for the Stokes problem, SIAM Journal on Numerical Analysis 44, (2006), no. 1, 322-348.

[ABW15] M. Ainsworth, G. R. Barrenechea, and A. Wachtel, Stabilization of high aspect ratio mixed finite elements for incompressible flow, SIAM Journal on Numerical Analysis 53 (2015), no. 2, 1107-1120.

[AC00] M. Ainsworth and P. Coggins, The stability of mixed hp-finite element methods for Stokes flow on high aspect ratio elements, SIAM J. Numer. Anal. 38 (2000), no. $5,1721-1761$.

[AKL08] T. Apel, T. Knopp, and G. Lube, Stabilized finite element methods with anisotropic mesh refinement for the Oseen problem, Appl. Numer. Math. $5 \mathbf{8}$ (2008), no. $12,1830-1843$.

[BB01] R. Becker and M. Braack, A finite element pressure gradient stabilization for the Stokes equations based on local projections, Calcolo 38 (2001), no. 4, 173199.

[BBF93] C. Baiocchi, F. Brezzi, and L.P. Franca, Virtual bubbles and Galerkin-leastsquares methods (Ga.L.S.), Comput. Methods Appl. Mech. Engrg., 105, (1993), no. 1, 125-141.

[BBF13] D. Boffi, F. Brezzi, and M. Fortin, Mixed finite element methods and applications, Springer Series in Computational Mathematics, vol. 44, Springer, Heidelberg, 2013. 
[BB+07] M. Braack, E. Burman, V. John, and G. Lube, Stabilized finite element methods for the generalized Oseen problem, Comput. Methods Appl. Mech. Engrg. 196 (2007), no. 4-6, 853-866.

[BDG06] P. B. Bochev, C. R. Dohrmann, and M. D. Gunzburger, Stabilization of loworder mixed finite elements for the Stokes equations, SIAM J. Numer. Anal. 44 (2006), no. 1, 82-101.

[BF01] F. Brezzi and M. Fortin, A minimal stabilisation procedure for mixed finite element methods, Numer. Math. 89 (2001), no. 3, 457-491.

[BH82] A. N. Brooks and T. J. R. Hughes, Streamline upwind/Petrov-Galerkin formulations for convection dominated flows with particular emphasis on the incompressible Navier-Stokes equations, Comput. Methods Appl. Mech. Engrg. 32 (1982), no. 1-3, 199-259.

[Bla08] J. Blasco, An anisotropic GLS-stabilized finite element method for incompressible flow problems, Comput. Methods Appl. Mech. Engrg. 197 (2008), no. $45-48,3712-3723$.

[BLR12] M. Braack, G. Lube, and L. Röhe, Divergence preserving interpolation on anisotropic quadrilateral meshes, Comput. Methods Appl. Math. 12 (2012), no. $2,123-138$.

[Bra08] M. Braack, A stabilized finite element scheme for the Navier-Stokes equations on quadrilateral anisotropic meshes, M2AN Math. Model. Numer. Anal. 42 (2008), no. 6, 903-924.

[Bur08] E. Burman, Pressure projection stabilizations for Galerkin approximations of Stokes' and Darcy's problem, Numerical Methods for Partial Differential Equations 24 (2008), no. 1, 127-143.

[BV10] G. R. Barrenechea, and F. Valentin, A residual local projection for the Oseen equation, Comput. Methods Appl. Mech. Engrg. 199, (2010), 1906-1921.

[BW15] G. R. Barrenechea and A. Wachtel, A note on the stabilised Q1-P0 method on quadrilaterals with high aspect ratios, Boundary and Interior Layers, Proceedings of BAIL 2014, P. Knobloch (Ed.), Lecture Notes in Computational Science and Engineering, Vol. 108, (2015), pp. 1-11.

[Cod08] R. Codina, Analysis of a stabilized finite element approximation of the Oseen equations using orthogonal subscales, Applied Numerical Mathematics 58, (2008), 264-283.

[CF03] Z.-H. Cao and L.-H. Feng, A note on variational Representation for singular values of matrix, Applied Mathematics and Computation, 143, (2003), 559563.

[FF92] L. P. Franca and S. Frey,, Stabilized finite element methods. II. The incompressible Navier-Stokes equation, Comput. Methods Appl. Mech. Engrg. 99 (1992), no. 2-3, 209-233. 
[FH88] L. P. Franca, and T. J. R. Hughes, Two classes of mixed finite element methods, Comput. Methods Appl. Mech. Engrg. 69 (1988), no. 1, 89-129.

[GL+12] K. Galvin, A. Linke, L. Rebholtz, and N. Wilson, Stabilizing poor mass conservation in incompressible flow problems with large irrotational forcing and application to thermal convection, Comput. Methods Appl. Mech. Engrg., 237, (2012), 166-176.

[GR86] V. Girault and P.-A. Raviart, Finite element methods for Navier Stokes equations, Springer, Berlin, 1986.

[HFB86] T. J. R. Hughes, L. P. Franca, and M. Balestra, A new finite element formulation for computational fluid dynamics. V. Circumventing the Babuška-Brezzi condition: a stable Petrov-Galerkin formulation of the Stokes problem accommodating equal-order interpolations, Comput. Methods Appl. Mech. Engrg. 59 (1986), no. 1, 85-99.

[HFH89] T. J. R. Hughes, L. P. Franca, and G. Hulbert, A new finite element formulation for computational fluid dynamics. VIII. The Galerkin/least-squares method for advective-diffusive equations, Comput. Methods Appl. Mech. Engrg. 73 (1989), no. 2, 173-189.

[Joh17] V. John, Finite element methods for incompressible flow problems, Springer Series in Computational Mathematics, vol. 51, Springer-Verlag, 2017.

[JJ+14] E. W. Jenkins, V. John, A. Linke, and L. G. Rebholz, On the parameter choice in grad-div stabilization for the Stokes equations, Adv. Comput. Math. 40 (2014), no. 2, 491-516.

[Kno10] P. Knobloch, A generalization of the local projection stabilization for convection-diffusion-reaction equations, SIAM J. Numer. Anal. 48 (2010), no. $2,659-680$.

[KS92] N. Kechkar and D. J. Silvester, Analysis of locally stabilized mixed finite element methods for the Stokes problem, Math. Comp. 58 (1992), no. 197, 1-10.

$[\mathrm{LM}+09]$ W. Layton, C. Manica, M. Neda, M.A. Olshanskii, and L. Rebholtz, On the accuracy of the rotation form in simulations of the Navier-Stokes equations, Journal of Computational Physics. 228 (2009), no. 9, 3433-3447.

[LS13] Q. Liao and D. Silvester, Robust stabilized Stokes approximation methods for highly stretched grids, IMA J. Numer. Anal. 33 (2013), no. 2, 413-431.

[MPP03] S. Micheletti, S. Perotto, and M. Picasso, Stabilized finite elements on anisotropic meshes: a priori error estimates for the advection-diffusion and the Stokes problems, SIAM J. Numer. Anal. 41 (2003), no. 3, 1131-1162.

[MST07] G. Matthies, P. Skrzypacz, and L. Tobiska, A unified convergence analysis for local projection stabilisations applied to the Oseen problem, M2AN Math. Model. Numer. Anal. 41 (2007), no. 4, 713-742. 
[MT15] G. Matthies and L. Tobiska, Local projection type stabilization applied to infsup stable discretizations of the Oseen problem, IMA J. Numer. Anal. 35 (2015), no. 1, 239-269.

[OR04] M.A. Olshanskii, and A. Reusken, Grad-div stabilization of the Stokes equations, Mathematics of Computation, 73, (2004), 1699- 1718.

[PS85] J. Pitkäranta and T. Saarinen, A multigrid version of a simple finite element method for the Stokes problem, Math. Comp. 45 (1985), no. 171, 1-14.

[RST08] H.-G. Roos, M. Stynes, and L. Tobiska, Robust numerical methods for singularly perturbed differential equations, second ed., Springer Series in Computational Mathematics, vol. 24, Springer-Verlag, Berlin, 2008. 